\title{
Screening for axon regeneration promoting compounds with human iPSC-derived motor neurons
}

Tammy Szu-Yu Ho ${ }^{1,2}$, J. Tabitha Hees ${ }^{1}$, Zhuqiu Xu¹, Riki Kawaguchi ${ }^{3}$ Natalia P. Biscola ${ }^{4}$, Daniel G Taub ${ }^{1,2}$, Kuchuan Chen ${ }^{1,2}$, Xirui Chen ${ }^{1}$, Lee B. Barrett ${ }^{1,2}$, Long Cheng ${ }^{1}$, Christopher V. Gabel ${ }^{5}$, Leif A. Havton ${ }^{6}$, Daniel H. Geschwind ${ }^{3}$,

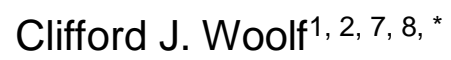

${ }^{1}$ F.M. Kirby Neurobiology Center, Program in Neurobiology, Boston Children's Hospital, Boston, MA 02115, USA. 2Department of Neurobiology, Harvard Medical School, Boston, MA 02115, USA. ${ }^{3}$ Program in Neurogenetics, Department of Neurology, David Geffen School of Medicine, University of California, Los Angeles, Los Angeles, CA 90095, USA. ${ }^{4}$ Department of Neurology, Icahn School of Medicine at Mount Sinai, New York, NY, 10029, USA. ${ }^{5}$ Department of Physiology \& Biophysics, Boston University Medical Center and Boston University Photonics Center, 700 Albany St, Boston, MA, 02118, USA. ${ }^{6}$ Departments of Neurology and Neuroscience, Icahn School of Medicine at Mount Sinai, New York, NY, 10029, USA. ${ }^{7}$ Harvard Stem Cell Institute, Harvard University, Cambridge, MA 02138, USA.

${ }^{8}$ Lead Contact

*Correspondence: $\underline{\text { Clifford.Woolf@childrens.harvard.edu }}$ 


\section{Summary}

CNS neurons do not regenerate after injury, leading to permanent functional deficits. Although sensory and motor neuron axons do regrow after peripheral nerve injury, functional outcome is limited due to the incomplete and slow regrowth. The lack of human-relevant assays suitable for large-scale drug screens has limited neuro-repair therapy discovery. To address this we developed a phenotypic screening strategy using human induced pluripotent stem cell-derived motor neurons to identify axon-regeneration promoting compounds and targets. The screens involve both re-plating human motor neurons on chondroitin sulfate proteoglycans and measuring regeneration responses to laser axotomy in spot cultures, and from them we identified multiple hits that promote injured axon regrowth. The top hit blebbistatin, a nonmuscle myosin II inhibitor, accelerated axon regeneration and functional recovery after sciatic nerve injury in vivo. Human "injury in a dish" assays are suitable, therefore, to screen for therapeutic interventions that can induce or accelerate axon regeneration.

\section{Keywords}

Axon regeneration, Human iPSC, Human-induced neurons, Screening 


\section{Introduction}

Neurons cannot regenerate after injury in the adult mammalian central nervous system (CNS) because of a combination of the failure to induce transcription of those genes that promote axon elongation and target innervation (Afshari et al., 2009; Giger et al., 2010; Mar et al., 2014; Renthal et al., 2020) and the presence of an extracellular and cellular environment not conducive to axonal growth (Fawcett, 2020; Filbin, 2003; Yiu and He, 2006). Although the axons of sensory and motor neurons do regenerate in the peripheral nervous system (PNS), motor functional recovery in patients with proximal nerve injury is minimal (Grinsell and Keating, 2014; Lundborg, 2000; Ma et al., 2011; Novak et al., 2011; Songcharoen et al., 2001), because of the slow pace of axonal regrowth after injury and the consequent delayed muscle re-innervation (Gordon et al., 2011; Hoke et al., 2002). CNS and PNS injuries affect millions and are devastating, yet currently, there is no approved drug to either induce regeneration of injured CNS neurons or accelerate regeneration in the PNS.

A lesion to the peripheral axons of dorsal root ganglion neurons activates intrinsic axonal growth-promoting genes (Chandran et al., 2016; Hoffman, 2010; Renthal et al., 2020), and as a consequence, a pre-conditioning peripheral nerve lesion both enhances regeneration of peripheral sensory axons (Jenq et al., 1988; McQuarrie et al., 1977) and enables regeneration of the central axons of sensory neurons in the dorsal columns beyond the lesion site in the spinal cord (Neumann and Woolf, 1999), indicating that a switch from a non-growth to an active axon growth state is a key element for both inducing regeneration in the CNS and enhancing it in the PNS. In addition, there is strong data 
supporting the benefit of either providing a growth-supportive and enhancing environment after nerve injury (David and Aguayo, 1981; Richardson et al., 1980) or suppressing an inhibitory one (Burnside et al., 2018; Hu et al., 2018; Schwab and Strittmatter, 2014; Tran et al., 2018; Wang et al., 2014). Although overexpressing key regeneration-driving genes can promote nerve regeneration (Fagoe et al., 2015; Ma et al., 2011; Seijffers et al., 2007), gene therapy is technically challenging and the question is whether it might be possible to promote regeneration and functional restoration after injury using small molecule drugs.

To develop drug-based therapies for promoting axon regeneration, robust human-relevant assays suitable for large scale drug screens are essential. One of the advantages of using models of human induced pluripotent stem cell (iPSC)-derived neurons is the feasibility to study both human biology and diseases and eliminate species differences. Compound screens have been performed on rodent neurons to look for molecules that enhance neurite outgrowth and promote regeneration (Al-Ali et al., 2015; Al-Ali et al., 2013; Huebner et al., 2019; Koprivica et al., 2005; Li et al., 2016; Ma et al., 2010; Usher et al., 2010), but this has not led to successful drug therapy. Modeling neurite outgrowth in human PSC-derived neurons is possible (Clarke et al., 2017; Sherman and Bang, 2018) and a screen to look for neurite growth modulating compounds in human iPSC-derived cortical-like neurons on PDL coated plates supplemented with laminin has been performed (Sherman and Bang, 2018). To mimic human neuron injury, a stretch injury model in a 96-well format using induced cortical-like neurons was developed (Sherman et al., 2016). However, there is currently no axotomy assay in human iPSC-derived 
neurons suitable for high-throughput screening.

Our goal here was both to develop a screening funnel that could identify targets that promote axon regeneration, by detecting the actions of unbiased bioactive target-annotated small molecules, and that has the high throughput characteristics necessary for future screens against large, chemically diverse libraries. Here we assessed the effect of bioactive small molecule targetannotated compounds on neurite outgrowth as a means of identifying new targets regulating regeneration, as well as compounds with potential for repurposing or drug development. Our strategy was to develop several different human iPSC-derived motor neuron regrowth phenotypic assays suitable for high-content screening. First, we screened for compounds that promote neurite outgrowth of re-plated motor neurons grown on chondroitin sulfate proteoglycans, a substrate selected to mimic an inhibitory extracellular matrix environment. For secondary screens, we both used laser injury of established axons in a human neuron spot culture assay and measured the dynamic outgrowth over days of live replated neurons after drug treatment. The final step of the screening funnel was to test the top hit in vivo in a mouse injury model.

From a screen of 4557 bioactive compounds we identified multiple novel hits whose action in promoting regeneration are reported here for the first time. We also found some hits consistent with previous studies in rodent models. The hit with greatest neurite outgrowth promotion in freshly dissociated neurons and regrowth of established axons after injury was blebbistatin, a non-muscle myosin II inhibitor that has been found before to promote neurite outgrowth in in vitro rodent primary neurons (Hur et al., 2011). Blebbistatin increased the 
neurite outgrowth of human induced motor neurons on CSPG more than 10fold. This effect is much greater than known growth promotors, like ROCK inhibitors (Chan et al., 2005; Fournier et al., 2003; Hiraga et al., 2006; Lingor et al., 2008). Furthermore, the growth promoting effects of blebbistatin in the human in vitro injury model predicted axon regeneration in a mouse in vivo injury model.

\section{Results}

\section{Phenotypic screen with human motor neurons to identify pro- regenerative compounds}

We designed a screening funnel to identify target-annotated compounds that promote axon regeneration using human-induced pluripotent stem cell (iPSC)derived motor neurons (Figure $1 \mathrm{~A}$ ). The primary screen, conducted with a bioactive compound library set, utilized a high-content neurite outgrowth promotion phenotypic assay. Dose response confirmation was performed on hits from the primary screen, followed by secondary screens utilizing an axonal injury and dynamic growth assay, followed by target validation by knockdown. Finally, we conducted in vivo studies using a mouse sciatic nerve injury model on the most promising hit to establish if the in vitro screen is predictive in revealing pro-regenerative targets for nerve repair.

The primary screen comprised determining axon growth 24 hours after replating motor neurons in wells on a substrate composed of chondroitin sulfate proteoglycans (CSPG) - a major component of the extracellular matrix in the central nervous system after injury (Yiu and $\mathrm{He}, 2006)$, to mimic regrowth in an 
injured environment. A positive control for the primary screen was laminin, a permissive substrate that promoted an increase in total neurite length per neuron of 6 -fold on average $(p<0.001)$ when compared with growth on CSPG (Figure 1C, D and G). A small molecule positive control for the screen was $Y$ 27632 a Rho-associated, coiled-coil-containing protein kinase (ROCK) inhibitor that promotes axon regeneration in rodent models (Chan et al., 2005; Fournier et al., 2003; Lingor et al., 2008). In pilot experiments we found that Y-27632 promotes outgrowth of re-plated human motor neurons (Figure 1B, E and F) with a 3.5-fold increase in average total neurite length per neuron at $20 \mu \mathrm{M}$ when compared with a DMSO control, $p<0.001$. Figure 1B shows the dose response curve for Y-27632. A 3D histogram of a 96-well screening plate from the primary screen with the positive (purple laminin and green Y27632) and negative controls (dark blue, CSPG and DMSO) is shown in Figure 1I. The middle columns (light blue) are growth responses to a set of small molecules from a bioactive library.

The overall strategy for this primary screen is shown in Figure 1J. Human motor neurons were differentiated from iPSCs utilizing a protocol with a series of small molecules: dual SMAD inhibition to neuralize the iPSCs, FGF and Notch signaling inhibition to accelerate neuronal differentiation, and activation of retinoic acid and Sonic Hedgehog signaling to pattern and specify motor neuronal fate (Klim et al., 2019). After the differentiation, the motor neurons underwent an axonal injury surrogate by re-plating them from the differentiation plate into a 96-well plate on CSPG. After treating neurons with compounds from the library for 24 hours, we identified those that promoted neurite outgrowth, using $\beta$ III-tubulin and Hoechst staining, with imaging and quantification with an 
Arrayscan (Figure 1J). To quantify neurite outgrowth induced after the re-plating, the average neurite length per neuron after compound treatment was normalized to the DMSO control.

We screened several bioactive small molecule libraries composed of targetannotated compounds (see Methods) to both identify pathways regulating regeneration and compounds with potential for drug development/repurposing. A total of 4557 compounds were screened and we observed hits that promoted neurite outgrowth (fold increase in neurite length $>1$ ) as well as hits that inhibited outgrowth (fold increase in neurite length<1). The top $1 \%$ of the growth positive hits had more than a 2-fold increase in neurite outgrowth when compared with the negative control, $0.15 \%$ DMSO. The primary screen results from the replated human motor neurons on CSPG are shown in a scatter plot, where each dot represents a small molecule (Figure $1 \mathrm{~K}$ ).

\section{Primary screen results reveal pro-regeneration target pathways.}

Table 1 lists the top 20 hits from the primary screen, of which blebbistatin, a non-muscle myosin II inhibitor, had the greatest axon growth promoting action. As shown in Figure $1 \mathrm{~K}$, blebbistatin increased the outgrowth of human motor neurons after re-plating 12-fold when compared with the DMSO control, which is substantially greater than both our positive controls (laminin and Y-27632 a ROCK inhibitor) and indeed all other hits. Figure $2 A$ and $B$ show images from a screening plate of neurons treated with blebbistatin that extend more neurites than the DMSO control, and Figure $2 \mathrm{C}$ shows the dose response curve of blebbistatin in the assay (red line indicates effect of $\mathrm{Y}-27632$ at $20 \mu \mathrm{M}$ ). Consistent with these findings, blebbistatin promotes neurite outgrowth in avian 
and rodent primary neurons and in human derived cortical-like interneurons (Hur et al., 2011; Rosner et al., 2007; Sherman and Bang, 2018). However, the drug has not been identified before in human neuron injury models.

The top 20 hits include several with an action on previously reported preregeneration pathways, indicating that the primary screen replicates other axon growth assays. This includes ROCK inhibitors, with five ROCK inhibitors amongst the top 20 hits, one of which was the positive control Y-27632. That ROCK inhibitors increase axon growth has been extensively studied (Chan et al., 2005; Fournier et al., 2003; Hiraga et al., 2006; Lingor et al., 2008). Among the ROCK inhibitors, RKI-1447 had the most potent effect on outgrowth promotion (Table 1 and Figure 2D). Recent reports reveal that RhoA, the immediate upstream target of ROCK, regulates cytoskeleton through nonmuscle myosin II, and that the physical target of RhoA in axon growth is nonmuscle myosin II (Dupraz et al., 2019). This suggests that ROCK inhibitors may promote axon regeneration by inhibiting non-muscle myosin II. Inhibiting nonmuscle myosin II directly, as blebbistatin does, could though, be more advantageous than inhibiting ROCK, which will also impact other effectors downstream of ROCK. Notably, we observed a much greater promotion of neurite outgrowth after treatment with blebbistatin, the non-muscle myosin II inhibitor, than with any of the five ROCK inhibitor hits.

Another group of top hits in the primary screen were voltage-gated calcium channel blockers, especially those with a vasodilator action. These include benidipine hydrochloride (dihydropyridine), an L-, T-, and N-type calcium channel blocker used for treating hypertension (Table 1), and nicardipine 
hydrochloride, also an L-type calcium channel blocker and antihypertensive drug, which promoted motor neuron neurite outgrowth 2.26-fold. We earlier found in primary rodent neurons, that the L-type calcium channel blocker diltiazem (benzothiazepine) promotes axon regeneration both in rat embryonic cortical neurons and adult primary mouse dorsal root ganglion neurons, as well as in induced human sensory neurons (Huebner et al., 2019).

Four JAK inhibitors were among the top 20 hits (Table 1) and the dose response curves of these are shown in Figure 2E, G and Supplementary Figure 1A and B. That four drugs from an unbiased compound library screen that act on the same target pathway were top hits, make JAK inhibition a compelling neurite growth promotion pathway.

AT7867 and CCT128930, both inhibitors of Akt1/2/3 and p70S6K/PKA, represent novel hits/targets for neurite outgrowth (Table 1; dose response curves of AT7867 and CCT128930 are shown in Figure 2F and H). To test which of the two pathways are responsible for the outgrowth promotion, we studied the individual pathways with more specific inhibitors. When motor neurons were treated with specific Akt inhibitors, no increase in neurite outgrowth was detected (Figure 2I). Although PKA inhibition did promote neurite outgrowth to some extent (Figure $2 \mathrm{~J}$ and $\mathrm{K}$ ), the effects were less potent than the combined inhibition of both Akt1/2/3 and PKA (Figure 2L). These data suggest that inhibition of both Akt1/2/3 and p70S6K/PKA is required for promoting human motor neuron axonal outgrowth. 
In the large-scale primary screen we treated human motor neurons with compounds for a fixed 24 hour period and recorded neurite growth at this time. To study dynamic drug effects of hits and for a longer duration, we developed a secondary assay where we tracked the responses of live motor neurons to the drug treatment using an IncuCyte S3 (Essen BioScience), which comprises an automated microscope inside an incubator, and this allowed us to trace neurites without a reporter or label and image and analyze neuron cultures over time (five days) such that we could measure real-time responses to drug treatment. Blebbistatin dramatically promoted neurite outgrowth over the 5 days of treatment when compared with DMSO control (Figure 3A, B and Supplementary movies 1 and 2). Figure 3C shows the effects of the top 10 hits from our primary screen on dynamic human motor neuron outgrowth after replating, at working doses established from the dose response curves.

\section{A human neuron in vitro injury assay to study axon regrowth}

The primary and secondary dynamic growth screens used the re-plating of differentiated neurons from a culture well as an injury surrogate. While this was efficient, reliable and robust, and therefore suitable for high-throughput primary screening, it is a harsh condition with the severing of all neuronal processes. To be closer to a patient injury situation, we developed a human neuron spot culture laser cutting assay (Figure 4A). We cultured a high density of human induced motor neurons as a dot or spot (120,000-150,000 cells per 1-7 $\mu \mathrm{l} / \mathrm{spot})$. Axons grow radially outward from the center of the spot (cell bodies) (Figure $4 G)$. After growing for 7 days, a defined set of long axons projecting in the same direction could then be severed by an infrared laser (Stiletto, Hamilton Thorne). The laser objective, together with an automated stage and the controller 
software, allowed us to target the axons and cut them precisely (Supplementary movie 3). This, together with IncuCyte imaging, enabled us to study live neuronal responses to an area of defined axonal injury in real time. Several hours after the laser axotomy distal axons start to degenerate and proximal axons begin to regrow (Figure 4B).

To study whether hits from the primary replating screen, which incorporates both neurite formation and axon elongation, also promote regeneration after axotomy, we applied hit compounds to the spot cultured neurons immediately after the laser cutting and observed axonal growth responses over time (Figure 4A). Blebbistatin significantly promoted regeneration after axonal injury in the human neuron spots (Supplementary movie 4 and 5). Figure 4C and D show the axon regrowth 40 hours after injury and drug treatment (DMSO in Figure 4C; blebbistatin in Figure 4D). Representative images show axon regrowth 40 hours after injury and drug treatment (Figure 4C-F). We quantified the degree of axon regrowth by a sholl analysis. Figure $4 \mathrm{H}$ shows the injury responses after treatment of the top 5 hits from the primary screen. In addition to blebbistatin, the other 4 tested hits also significantly promoted regeneration of human motor neurons after axonal injury (Figure $4 \mathrm{H}$ ) indicating that the primary screen did detect axon regeneration promoting hits.

The spot culture injury assay is not only suitable as a secondary validation assay but is also amenable for screening. We adapted the assay so that human motor neurons are plated as one spot in each well of a 96-well plate (Figure 4A; examples in Supplementary movie 6 and 7). 


\section{Target Validation}

Knock down of non-muscle myosin II, the target of blebbistatin, with NMIIA shRNAs promoted axon regrowth after injury in the human motor neuron spot culture (Supplementary Figure 2A and B), confirming that inhibition of the annotated target of blebbistatin is responsible for its action. A role for nonmuscle myosin inhibition on regeneration was also detected in Caenorhabditis elegans (Supplementary Figure 2C) where knocking down nmy-1 significantly enhanced axon regeneration in $C$. elegans after laser cutting axons in vivo indicating that this is a general cross-species effect.

\section{Genes changed after blebbistatin treatment are linked with regeneration- associated genes.}

Blebbistatin, as a non-muscle myosin II inhibitor reorganizes actin and microtubules in growth cones, resulting in rapid axon extension (Dupraz et al., 2019; Hur et al., 2011). Although there are many actin and microtubule regulators in the compound libraries we screened, none had such a dramatic effect in promoting neurite outgrowth as blebbistatin. We therefore hypothesized that blebbistatin may play an additional role beyond regulating actin cytoskeleton at the growth cone. Inhibition of non-muscle myosin II by blebbistatin changes gene expression in human pluripotent stem cells (Walker et al., 2010), possibly through changes in actin-myosin based contractile forces which convert to changes in signaling. To test if a blebbistatin mediated alteration in gene expression may be involved in promoting regeneration in our assays, we collected dissociated human motor neurons at different time points after re-plating on CSPG and treating with blebbistatin or a DMSO control (16, 26 and 48 hours after drug treatment). Supplementary Table 1 shows the top 
20 differentially expressed genes significantly upregulated after blebbistatin treatment for 16 hours $(F D R<0.1)$. The most up-regulated differentially expressed gene is MMP-7, matrix metallopeptidase 7 , with $>16$-fold increase in expression after blebbistatin treatment when compared with DMSO (logFC = log2 Fold Change). Some known regeneration-associated genes (RAG) are also upregulated after blebbistatin treatment, including IFITM2, one of the top 10 upregulated differentially expressed genes (Supplementary Table 1). BMP6 expression also increased after blebbistatin treatment. BMP signaling can activate a pro-regenerative transcriptional program (Zhong and Zou, 2014).

A gene ontology analysis of those genes significantly up-regulated in blebbistatin-treated human motor neurons reveals several enriched biological processes (Figure 5A-C) and molecular functions (Supplementary Figure 3AC), including extracellular matrix disassembly/organization, chemokine activity and microtubule motor activity. We compared the differentially expressed genes after blebbistatin treatment with the well-characterized regeneration-associated gene (RAG) set involved in axon regeneration after injury (the magenta module in Chandran et al., 2016). Blebbistatin responsive genes significantly overlap with RAGs (Hypergeometric test, P-value=1.12 x 10-8, $5.38 \times 10^{-11}$ and $3.16 \times$ 10-11 for 16, 26, 48 hour treatments, respectively) (Figure 5D-F). Some upregulated RAGs induced in motor neurons after blebbistatin treatment are shown as a heatmap in Figure 5G. Gene expression changes after blebbistatin treatment are linked, therefore, with RAGs, and these may be involved, together with cytoskeletal changes in the growth cone, in increasing axon regrowth after blebbistatin treatment. 


\section{Blebbistatin promotes axon regeneration and functional recovery after} sciatic nerve injury in vivo.

To determine whether blebbistatin promotes axon regeneration after injury in vivo, we used a mouse sciatic nerve transection model. After transecting the sciatic nerve, the two nerve ends were bridged with a $5 \mathrm{~mm}$ silicon tube (Figure $6 \mathrm{~A})$ and the extent and timing of regrowth across the bridge measured. Between the two nerve endings we left a $3 \mathrm{~mm}$ gap and filled this with hydrogel mixed with either blebbistatin or vehicle, to locally treat the injured axons (Figure 6A and B), given that blebbistatin has poor solubility, inhibits muscle myosin II as well as non-muscle myosin II, is unstable and not well suited for systemic exposure (Dou et al., 2007; Eddinger et al., 2007; Limouze et al., 2004; Roman et al., 2018; Straight et al., 2003; Wang et al., 2008; Young et al., 2016). The nerves after regrowth were dissected at different time points, cryosectioned and immunostained with NF-200 antibody to label regenerated axons. Two weeks after injury and drug treatment, the blebbistatin treated group had more axons that had regrown through the gap region than the vehicle treated group (Figure 6C and D). Eight weeks after injury and drug treatment, the axons in the vehicle control group also grew through the gap but to a lesser extent (Figure 6F and G). A sholl analysis of axon numbers confirmed that blebbistatin significantly promoted axon regeneration (Figure 6E).

To study whether the regenerated axons remyelinated we performed light microscopy (LM) and electron microscopy (EM) on transverse sections of the regrown axons in the tube 8 weeks after the injury and blebbistatin treatment. The regenerated axons were myelinated 8 weeks after the injury both when treated with blebbistatin (Figure $6 \mathrm{H} \& \mathrm{I}$ ) and with vehicle control (Figure 6J \& $\mathrm{K}$ ) 
but the blebbistatin-treated group had significantly more myelinated axons then the vehicle-treated group (Figure 6M) $(P$-value=0.00069, unpaired two-tailed $t$ test). The newly regenerated myelinated axons tended to form multiple distinct bundles, as shown in by EM (Figure $6 \mathrm{~N}$ ).

We also studied functional motor recovery of the blebbistatin treated mice using a DigiGait (Mouse Specifics, Inc.) to image recovery of paw spread of the mice while walking on a treadmill (Fig. 6L). After sciatic nerve transection, the hind paw on the injured side does not spread out normally (Fig. 6L, red square in the vehicle group), while the hind paw of the sham control non-injured group shows normal paw spreading (Fig. 6L, red square in the sham control group). Blebbistatin treatment improved paw spreading (Fig. 6L, red square in the blebbistatin group). Quantification of the sciatic nerve function index showed that blebbistatin improved motor functional recovery starting 6 weeks after injury (Fig. 60).

Taken together, these data show that blebbistatin, the top hit in the human motor neuron pro-regenerative primary and secondary screens, promotes axon regeneration and functional motor recovery after sciatic nerve injury in vivo and that these screens can be used to find accelerators of peripheral axonal regrowth. 


\section{Discussion}

Our goal here was to develop in vitro human cell-based assays suitable for efficient screening in an unbiased and high throughput fashion for novel ways of promoting axon regeneration with therapeutic potential.

\section{Implications of the primary screening strategy.}

The human iPSC-derived motor neuron growth assay we developed for the primary screen comprises measuring neurite growth over a fixed period (24 hours) after re-plating differentiated motor neurons onto a CSPG substrate. The re-plating of the neurons effectively severs all their axons from the cell body and the growth that is then seen includes both initial neurite formation and then their elongation on the inhibitory substrate and is both consistent $\left(R^{2}=0.96\right.$ between replicates) and reliable ( $Z^{\prime}$ factor $>0.5$ ). While use of CSPG substrates mimics aspects of the inhibitory CNS environment (Yiu and $\mathrm{He}, 2006$ ), it also provides a larger window to observe pro-regeneration drug effects than if the cells are plated on a permissive substrate, like laminin, as tested in pilot studies, improving the Z'.

The libraries we used for the screening comprise target-annotated bioactive compound sets, allowing us not only to identify compound hits but also targets, such as non-muscle myosin II, JAK and Akt1/2/3 and p70S6K/PKA. Involvement of non-muscle myosin II as an outgrowth suppressor target was validated by target knockdown which phenocopied the inhibitor.

Since we performed the screen in human motor neurons, one question is 
whether the pro-regenerative targets we identified may be applied to other neurons. To address this, we tested the effect of blebbistatin on a different neuronal type. Consistent with the effect on human motor neurons, blebbistatin also promotes outgrowth of human iPSC-derived sensory neurons (Supplementary Figure 1C), indicating the effect is not specific to motor neurons.

\section{Blebbistatin treatment changes gene expression in human motor neurons.}

Since blebbistatin caused such dramatic effects both on neurite outgrowth and axon regrowth (Figure. 2-4), we wondered if in addition to regulating the cytoskeleton at the growth cone, blebbistatin also changed gene expression, turning on genes involved in axon regeneration? To study blebbistatin induced transcriptional changes we collected human induced motor neurons after treating with blebbistatin at various time points and performed RNA Seq analysis. The top up-regulated differentially expressed gene after blebbistatin treatment for 16 hours, was matrix metallopeptidase 7 (MMP-7), with $>16$ fold increase in expression. MMP-7 is a secreted protease that cleaves extracellular matrix (ECM) components including CSPG (Wang et al., 2018). MMP-7 transcripts are upregulated 24 hours after a spinal cord injury in mice (Wells et al., 2003) and MMP-7 is proposed to have a neuroprotective effect through cleavage of pro-neurotrophins (Le and Friedman, 2012; Lee et al., 2001).

In addition to ECM components, and genes involved in organizing ECM molecules, genes that regulate inflammation and immunity were also upregulated (Supplementary Table 1). CXCL5 is a cytokine that has neurotrophic effects and promotes neurite growth of rat pelvic ganglia (Zhang et al., 2011). CXCL5 binds to the chemokine receptor CXCR2, which is 
expressed by human iPSC-induced motor neurons as well as mouse DRG neurons (http://www.painseq.com) and the signaling induced by CXCL5 might be involved in axon regrowth.

\section{Blebbistatin promotes axon regeneration in vivo}

Blebbistatin significantly promoted regeneration after axonal injury in the human motor neuron spot axotomy model (Figure. 4D and $\mathrm{H}$ ). Furthermore, blebbistatin also promoted regeneration after nerve injury in vivo in the adult mouse (Figure. 6D, E, F). In the sciatic nerve transection and bridge repair model, we can treat the injured nerve locally by mixing the drug with hydrogel and applying it into to the gap in the bridge between the proximal and distal parts of the nerve (Figure. 6A). Remyelination and formation of nodes of Ranvier were observed on regenerated axons crossing the gap indicating that not only was axon growth promoted but interactions with Schwann cells occur (Figure. 6H-M and data not shown). Furthermore, blebbistatin not only enhanced axon regrowth but also promoted a measurable increase in functional motor recovery after sciatic nerve injury (Figure. 60).

While blebbistatin produces dramatic effects on axon regrowth, it is not an ideal drug for therapeutic use. It also inhibits cardiac-, skeletal-, and smooth-muscle myosin II (Dou et al., 2007; Eddinger et al., 2007; Limouze et al., 2004; Straight et al., 2003; Wang et al., 2008), has poor water solubility and poor microsomal stability (Roman et al., 2018; Young et al., 2016). Nevertheless, mice given systemic blebbistatin by intraperitoneal injection show normal behavior including locomotion and other general health measures (Young et al., 2016). In addition, blebbistatin crosses the blood brain barrier (Young et al., 2016) and 
could be suitable to explore promotion of CNS regeneration. Although modifications have been made to improve certain properties of blebbistatin, including solubility and photochemical features (Kepiro et al., 2014; Roman et al., 2018; Varkuti et al., 2016), a MedChem project to generate more specific non-muscle myosin II inhibitors with high potency and stability and desirable drug-like properties are required to fully explore the pro-regenerative therapeutic opportunities of this target. Utilization of the human axon outgrowth models described here, would assist such a MedChem drug development program.

\section{Limitations of study}

There are some limitations to our primary screen, for example, we used a fixed concentration of the 4557 compounds $(10 \mu \mathrm{M}$, except for the Biomol library where concentrations vary, based upon potency and availability). Therefore, we may miss candidates whose working doses are at a different range. To address this, compound libraries could be screened at multiple concentrations, although there is always a tension between more compounds or more concentrations. We chose to conduct a full dose response analyses only on the hits.

Another limitation of the screen was that the iPSC derived motor neurons are relatively immature. Therefore, we validated the top hit in adult mice to establish if the effects were pro-regenerative rather than developmental growthpromoting, but it would be ideal to be able do this in vitro. In addition to the short-term neurite outgrowth promotion detected by the primary screen, we performed secondary assays that were designed to study dynamic drug effects over days and whether responses after axonal injury are similar to that after cell 
dissociation. Because each assay detects different aspects of the growth response and constitute independent replication of axon growth promotion actions, we feel that replication in both is informative of action on axon growth.

In conclusion, phenotypic screens of human motor neurons for axon outgrowth and regrowth, can be used for the development of treatments for the repair of the injured peripheral nervous system.

\section{Acknowledgments}

This work was funded by the NIH (R35NS105076, C.J.W.), the Dr. Miriam and Sheldon G Adelson Medical Research Foundation (L.A.H., D.H.G. and C.J.W.), a Leonard and Isabelle Goldenson fellowship and an Ellen R. and Melvin J. Gordon fellowship (T.S-Y.H.). Figures 1J,4A, and 6A were made using BioRender.

We are grateful to Zigang He for discussions about this project and to our colleagues Andrew Snavely, Bhagat Singh, Yung-Chih Cheng, Laurel Heckman and Xuan Huang who provided expertise and advice that greatly assisted the research. We express our gratitude to Jennifer Smith and the ICCB-Longwood Screening Facility at Harvard Medical School.

\section{Author contributions}

Conceptualization, T.S-Y.H. and C.J.W.; methodology, T.S-Y.H., T.H., Z.X.; validation, T.S-Y.H., T.H., Z.X., N.P.B., D.G.T., and X.C.; formal analysis, T.SY.H. and R.K.; investigation, T.S-Y.H., T.H., Z.X., N.P.B., D.G.T., X.C., K.C., 
H.A.J.; resources, L.B.B., C.V.G., L.A.H., D.H.G. and C.J.W.; original draft, T.S-Y.H. and C.J.W.; supervision, T.S-Y.H., L.A.H., D.H.G. and C.J.W.

\section{Declaration of interests}

No conflicting interests 


\section{Figure legends}

Figure 1. High-throughput phenotypic screen in human iPSC-derived motor neurons to identify pro-regenerative compounds.

(A) Screening funnel illustrating the screening strategy. (B) Dose response curve of $\mathrm{Y}-27632$, a positive control for the primary screen on neurite outgrowth (total neurite length per neuron). (C-D) Human iPSC-derived motor neurons cultured on laminin, a positive control substrate (C), or CSPG (D) for 24 hours after re-plating. Neurons stained with an antibody against $\beta$ III-tubulin. Scale bar, $50 \mu m .(E-F)$ Human motor neurons treated with Y-27632 (positive control, E) or DMSO (negative control, F) for 24 hours after re-plating on the CSPG substrate.

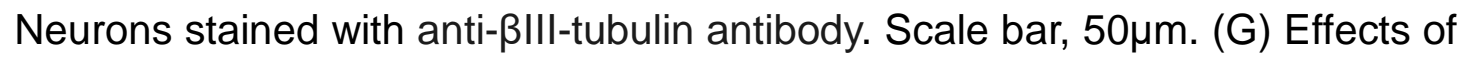
laminin vs CSPG on neurite outgrowth 24 hours after re-plating. ( ${ }^{\star \star *} \mathrm{P}=0.000056$, unpaired two-tailed t-test, $\mathrm{n}=5$ for each group). $(\mathrm{H})$ Number of neurons on laminin vs CSPG fixed, stained and quantified 24 hours after replating. ( ${ }^{\star \star} \mathrm{P}=0.002$, unpaired two-tailed t-test, $\mathrm{n}=5$ for each group). (I) A 3D histogram of a 96-well screening plate with positive (purple, laminin; green, Y27632) and negative (dark blue, DMSO) controls. Test compounds (light blue) are in columns 2-11. $\mathrm{Z}$ axis shows total neurite length per neuron after compound treatment for 24 hours. (J) Primary screening strategy: Motor neurons were differentiated from human iPSC as illustrated. After differentiation, the neurons were re-plated on CSPG coated 96-well plates, treated with compounds for 24 hours, fixed, stained and imaged using the ArrayScan Screening System. (K) Primary screen results from 4557 compounds. Each dot represents a molecule. Data represented as mean \pm SD. 
Figure 2. Dose response curves of top hits from primary screen.

(A-B) Human motor neurons treated with $25 \mu \mathrm{M}$ blebbistatin $(\mathrm{A})$ or $0.15 \%$ DMSO negative control (B) for 24 hours after re-plating on CSPG. Scale bar, 100 $\mathrm{mm}$. (C-H) Dose response curves of top 6 hits: blebbistatin (C), RKI-1447 (D), CEP$33779(E)$, AT-7867 (F), Momelotinib (G), and CCT128930 (H), showing neurite outgrowth promotion after 24 hour treatment. (I-K) Dose response curves of an Akt inhibitor, MK-2206 (I) and PKA inhibitors, HA-1004 (J) and H9 (K). (L) Combinatorial treatment of Akt and PKA inhibitors shows better outgrowth enhancement than treatment with single inhibitors. $\left({ }^{\star \star \star} \mathrm{P}=0.0001,{ }^{\star \star} \mathrm{P}=0.0028\right.$, unpaired two-tailed t-test, $n=4$ for each group). Data represented as mean \pm SD.

Figure 3. Dynamic changes in human motor neurons produced by hits.

(A-B) Images of live human motor neuron taken at Day 1, Day 2 and Day 5 after $25 \mu \mathrm{M}$ blebbistatin treatment (A) or DMSO treatment (B). Scale bar, $100 \mu \mathrm{m}$. (C) Quantification of neurite length after drug treatment over time. The top 10 hits from the primary screen were used in the dynamic re-plating assay, neurons were imaged and analyzed every 6 hours over 5 days. Data represented as mean \pm SEM

Figure 4. Axon regeneration in an injured human neuron spot culture model.

(A) Flow chart of the human neuron spot injury assay. Human iPSC derived motor neurons were plated at high density as a spot (150,000 cells/spot). After growing for 1 week, axons were cut with laser at a specified area, and regrowth quantified with an IncuCyte. (B) Example of the response of human motor 
neurons to laser axotomy, red line indicates laser cutting site. Cell bodies were located below the images. Distal axons started to degenerate a few hours after

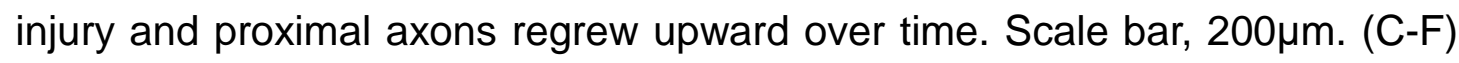
Representative images of laser axotomized spots treated with DMSO (C), blebbistatin (D), RKI-1447 (E), and AT7867 (F) at time 0 and 40 hours after axotomy. Scale bar, $200 \mu \mathrm{m}$. (G) Example of laser injured spot. Scale bar, $1 \mathrm{~mm}$. (H) Quantification of degree of regrowth using sholl analysis, showing regeneration response after injury and effects of treatment with top 5 hits from the primary screen. Data represented as mean \pm SEM. $(P=1.45 E-13,0.0084$, 0.016, $0.00003,0.0246$ for blebbistatin, RKI-1447, AT7867, Momelotinib, CEP33779, respectively, unpaired two-tailed t-test).

Figure 5. Effects of blebbistatin on transcriptional profiles of human motor neurons.

(A-C) Gene Ontology enrichment scores of overlapping up-regulated genes 16 (A), 26 (B), and 48 hours (C) after blebbistatin or DMSO treatment. (D-F) Venn diagrams showing overlapping genes between blebbistatin vs DMSO and a previously defined set of regeneration associated genes (Magenta) (Chandran et al., 2016) at 16 (D), 26 (E) and 48 hours (F) after treatment. (G) A heatmap of some up-regulated regeneration associated genes after blebbistatin treatment. See also Figure S3.

Figure 6. Blebbistatin promotes axon regeneration and functional recovery in an in vivo sciatic nerve injury model.

(A) Sciatic nerve transection and bridge repair model. (B) Drug treatment (hydrogel + blebbistain or hydrogel + vehicle) in $3 \mathrm{~mm}$ gap between nerve 
endings and results 2 weeks after injury. (C-G) Sciatic nerve sections 2 (C-D) or 8 weeks (F-G) after injury, stained with Neurofilament-200 antibody to label

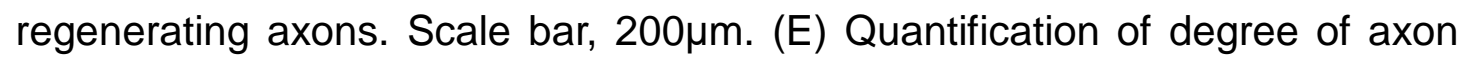
regeneration by sholl analysis. Data represented as mean \pm SEM. ( ${ }^{\star \star} \mathrm{P}$-value $=$ 0.009 for 4 weeks, ${ }^{\star}$ P-value $=0.0158$ for 2 weeks and 0.0152 for 8 weeks, unpaired two-tailed $t$-test). ( $\mathrm{H}-\mathrm{K})$ Images of representative toluidine bluestained cross sections of nerve and segmented axons for both blebbistatin and vehicle groups. Scale bar: $\mathrm{H}, \mathrm{J}: 50 \mu \mathrm{m}$. (M) Note larger number of fibers in the blebbistatin group than the vehicle group $\left({ }^{\star \star \star} p=0.0007\right)$. (N) Representative electron micrograph from Blebbistatin group showing clusters of myelinated fibers. Note varied size and degree of myelination between individual fibers. (L) Digigait images of mice 8 weeks after injury and drug treatment. Red squares label paws on injured side. (O) Quantification of motor function recovery by sciatic nerve functional index of 3 groups (blebbistatin-treated, vehicle-treated, and sham) 2, 4, 6, 8 weeks after injury. Data represented as mean \pm SD. ( ${ }^{*} \mathrm{P}=0.034$ for 6 weeks and ${ }^{*} \mathrm{P}=0.015$ for 8 weeks, unpaired two-tailed t-test). 
Table 1: Top 20 hits from the primary screen

\begin{tabular}{|c|c|c|c|}
\hline & Compound Names & Function / Target & Length \\
\hline 1 & Blebbistatin & Inhibitor of non-muscle myosin II ATPases & 12.00 \\
\hline 2 & RKI-1447 & Inhibitor of ROCK1/ROCK2 & 6.26 \\
\hline 3 & CEP-33779 & JAK2 inhibitor & 5.52 \\
\hline 4 & AT7867 & Inhibitor of $A k t 1 / 2 / 3$ and p70S6K/PKA & 4.33 \\
\hline 5 & Momelotinib & JAK1/2 inhibitor & 3.17 \\
\hline 6 & ССТ128930 & Inhibitor of Akt2 and p70 S6K/PKA & 3.09 \\
\hline 7 & Thiazovivin & Inhibitor of ROCK1/ROCK2 & 3.04 \\
\hline 8 & Y-27632 & Inhibitor of ROCK1/ROCK2 & 2.88 \\
\hline 9 & Benidipine hydrochloride & Voltage-gated L-type calcium channel blocker & 2.87 \\
\hline 10 & HA1077 (Fasudil) & $\begin{array}{l}\text { Inhibitor of ROCK1/2, less potent inhibition } \\
\text { over PKA, PKG, PKC }\end{array}$ & 2.66 \\
\hline 11 & Oxiconazole Nitrate & Ergosterol biosynthesis inhibitor & 2.62 \\
\hline 12 & Baricitinib & JAK1/2 inhibitor & 2.61 \\
\hline 13 & Lynestrenol & Agonist of the progesterone receptor & 2.56 \\
\hline 14 & Y-33075 & Inhibitor of ROCK1/ROCK2 & 2.55 \\
\hline 15 & Ruxolitinib & JAK1/2 inhibitor & 2.50 \\
\hline 16 & PD-176252 & Antagonist of BB1 and BB2 receptors & 2.45 \\
\hline 17 & Tribenoside & stimulates laminin $\alpha 5$ production & 2.40 \\
\hline 18 & Forodesine & Inhibitor of purine nucleoside phosphorylase & 2.38 \\
\hline 19 & LY2801653 & Inhibitor of MET tyrosine kinase & 2.35 \\
\hline 20 & RAF265 (CHIR-265) & Inhibitor of C-Raf/B-Raf/B-Raf V600E & 2.30 \\
\hline
\end{tabular}




\section{Materials and methods}

\section{Human iPSC culture}

iPSCs were maintained in StemFlex medium (Thermo Fisher) on culture dishes coated with Matrigel (Corning). Cells were passaged when at approximately 80\% confluence, using ReLeSR (Stem Cell Technologies) enzyme free passaging reagent. After 10 passages, a new vial of iPSCs was thawed. Human iPSC line SAH-0047 was provided by the Sahin laboratory (Boston Children's Hospital). Human iPSC line 11a was provided by the Eggan lab (Harvard University) (Boulting et al., 2011). SAH-004 was used for the primary screen. Both SAH-004 and 11a lines were used for secondary assays and showed consistent and reproducible results.

\section{Motor neuron differentiation from human iPSC}

iPSCs were differentiated into motor neurons as described (Klim et al., 2019) and illustrated in Figure 1J. In brief, iPSCs were dissociated into single cells using Accutase (Stem Cell Technologies) and plated at a density of 40,000 cells per $\mathrm{cm}^{2}$ in a $10 \mathrm{~cm}$ Matrigel (Corning) coated dish in StemFlex (Thermo Fisher) media with $10 \mu \mathrm{M}$ Y-27632 (Tocris) on day -2 . The next day, the medium was replaced by StemFlex medium without Y-27632. From day 0-5 (differentiation phase 1$)$, the medium was changed to differentiation medium (1/2 NeurobasalA (Thermo Fisher) $1 / 2$ DMEM-F12 (Thermo Fisher) supplemented with $\times 1$ B-27 supplement (Thermo Fisher), ×1 N-2 supplement (Thermo Fisher), ×1 Gibco GlutaMAX (Thermo Fisher) and $\times 1$ non-essential amino-acids (Thermo Fisher)) supplemented with $1 \mu \mathrm{M}$ retinoic acid (Sigma-Aldrich), $1 \mu \mathrm{M}$ SAG (Cayman Chemical), $100 \mathrm{nM}$ LDN-163189 (Sigma-Aldrich) and $10 \mu \mathrm{M}$ SB431542 
(Cayman Chemical). In phase 1, neural induction occurred with dual SMAD inhibition (SB431542 and LDN). Retinoic acid and SAG patterned the motor neuron fate. From day 6 - 13 (differentiation phase 2 ), the same differentiation medium was added, however, supplemented with $1 \mu \mathrm{M}$ retinoic acid, $1 \mu \mathrm{M}$ SAG, $4 \mu \mathrm{M}$ SU-5402 (Sigma-Aldrich) and $5 \mu \mathrm{M}$ DAPT (Tocris). SU-5402 and DAPT specified motor neuron identity.

On day 14, iPSC-derived motor neurons were dissociated with Accutase for 45 minutes at $37{ }^{\circ} \mathrm{C}$, sorted by magnetic-activated cell sorting (MACS) with antibody against human CD56 neural cell adhesion molecule 1 (BD Biosciences) and anti-R-phycoerythrin (PE) magnetic particles - DM (BD Biosciences), and then re-plated on desired plates for various studies. For primary screen, 8000 neurons were seeded in each well of CSPG $(0.2 \mathrm{ng} / \mathrm{ml}$, Millipore Sigma)-coated Corning BioCoat 96-well plates (Thermo Fisher). Human induced motor neurons were then cultured in motor neuron culture media (Neurobasal-A with N2 (1:100)/ B27 (1:50)/ Glutamax (1:100)/ ×1 NEAA (1:100)/penicillin-streptomycin (1:100)) supplemented with growth factors (35 $\mathrm{gg} / \mathrm{mL}$ ascorbic acid (Sigma), 10ng/mL recombinant human BDNF (Thermo Fisher), $10 \mathrm{ng} / \mathrm{mL}$ recombinant human GDNF (Thermo Fisher), $10 \mathrm{ng} / \mathrm{mL}$ recombinant human CNTF (Thermo Fisher)).

\section{Compound library}

The compound libraries used for the primary screen were provided by ICCBLongwood Screening Facility (Harvard Medical School). The small molecule libraries comprised known bioactives, including Biomol ICCBL Known Bioactives 2012 Library, Mechanism of Action Library, Prestwick 3 collection, 
and Selleck bioactive library. The drug concentration used for the primary screen was $15 \mu \mathrm{M}$ except for Biomol library, where compound concentration varied up to $0.1 \mathrm{mM}$ based upon potency and availability.

\section{Neurite outgrowth analysis - Primary Screen}

Dissociated human iPSC-derived motor neurons were re-plated on poly-D-

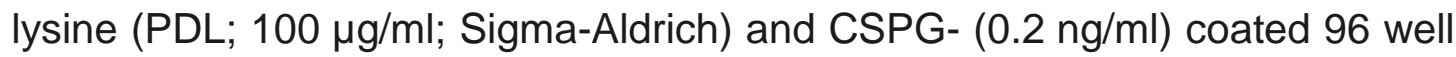
plates at a density of 8000 cells/well and treated with compound libraries. After neurons were cultured and treated with compounds for 24 hours, neurons were fixed with $4 \%$ paraformaldehyde (Electron Microscopy Sciences) and stained using a mouse antibody against BIII-tubulin (Sigma) and Hoechst 33342 (Thermo Fisher) for nuclear staining. Imaging and neurite outgrowth analysis were performed by a Cellomics ArrayScan XTI High-Content Screening System (Thermo Fisher). Images were acquired with a 10x objective with ArrayScan high-resolution camera. Data including valid neuron count, cell body area, total/individual neurite length, total neurite length per neuron, number of branches, etc. were collected and analyzed with the "Neuronal profiling" application. All results were normalized to DMSO control. Dose-response validation: At least 6 doses of each test compound were prepared with serial dilutions (ranging from $\mathrm{nM}$ to $\mu \mathrm{M}$ covering the working dose from the primary screen). For each compound concentration, four replicate wells were assayed. Cells were incubated in test compounds for 24 hours and then fixed with $4 \%$ paraformaldehyde. After staining with mouse anti-ßIII-tubulin antibody and Hoechst, total neurite length per neuron and valid neuron number were analyzed using ArrayScan XTI. 


\section{Human neuron spot culture}

High density $(150,000$ cells per spot) of human iPSC-induced neurons were plated on completely dry PDL- $(100 \mu \mathrm{g} / \mathrm{ml})$ and laminin- $(10 \mu \mathrm{g} / \mathrm{ml}$; Thermo Fisher) coated 96-well or 6-well plates. For each spot, $1 \mu \mathrm{l}$ cell suspension at a concentration of 150,000 cells/ $\mu$ l was added to the center of each well of 96 well plates or $6 \mu \mathrm{l}$ cell suspension of 150,000 cells to each well of 6 -well plates. The $1 \mu$ spots were kept in a humidity chamber in a $37^{\circ} \mathrm{C}$ incubator for 10 minutes (or 30 minutes for $6 \mu$ spots) to settle down and attach to the plates. Then the motor neuron culture media with growth factors was slowly and carefully filled into the wells without disturbing the spots $(100 \mu \mathrm{l}$ per well for $96-$ well plates and $2 \mathrm{ml}$ per well for 6 -well plates). Half medium was refreshed every 3 days. The axons grew outward radially from the spot (cell bodies) and extended continuously over time.

\section{Axon Injury with laser and regeneration analysis in human neuron spots}

The spots were cultured for 7 days to allow motor neurons to extend long axons before a laser axotomy with a $300 \mathrm{~mW}$ Stiletto infrared laser (Hamilton Thorne) combined with a 20x objective. The laser module has a high-speed micro controller and an automated motorized stage. Axons were cut (as illustrated in Figure 4G) $400 \mu \mathrm{m}$ away from the cell bodies at the edge of the spot. The spots were imaged with an IncuCyte S3 Live-Cell Analysis System (Sartorius) every 3-5 hours to record the dynamic regrowth of axons. The degree of axon regeneration was analyzed using a Sholl analysis (Fiji Is Just ImageJ) (Schindelin et al., 2012). The sholl analysis quantified the sum of intersections of regenerated axons and concentric circles. The images taken right after cut ( 0 hour) were used to determine the starting point for the sholl analysis. All 
results were normalized to a control DMSO-treated group.

\section{RNA-seq library preparation and sequencing}

Dissociated human motor neurons treated with blebbistatin or DMSO were harvested at different time points (16 hours, 26 hours and 48 hours) after replating and drug treatment. Total RNAs were extracted from these samples using Buffer RLT Plus (Qiagen), and then purified using RNeasy Plus mini kit (Qiagen). RNA-sequencing was carried out by HiSeq4000. Library was prepared using TruSeq Stranded RNA (100 ng) + RiboZero Gold. Quality control (QC) was performed on base qualities and nucleotide composition of sequences, to identify problems in library preparation or sequencing. Reads were trimmed and filtered, if necessary, after the QC before input to the alignment stage. Reads were aligned to the latest human GRCh38 reference genome using the STAR (v2.4.0) spliced read aligner. Average input read counts were $66.7 \mathrm{M}$ per sample (range 51.7 to $85.3 \mathrm{M}$ ) and average percentage of uniquely aligned reads was $90.5 \%$ (range $88.03 \%$ to $92.0 \%$ ). Total counts of read-fragments aligned to known gene regions within the human GRCh38 refSeq reference annotation were used as the basis for quantification of gene expression. Fragment counts were derived using HTS-seq (v.0.6.0) program using GRCh38 refSeq genes as model. Low count transcripts were filtered, and count data were normalized using the method of trimmed mean of M-values (TMM). Differentially expressed genes $($ FDR $<0.1)$ were then identified using the Bioconductor package EdgeR using paired-analysis (Robinson et al., 2010). The scripts that used in the RNA sequencing analyses are available at https://github.com/icnn/RNAseq-PIPELINE.git. 


\section{Mice}

10-week-old C57BL/6J mice were obtained from Jackson Laboratory (Bar Harbor). Animals were housed and handled in accordance with protocols approved by the institutional animal care and use committee (IACUC) of Boston Children's Hospital.

\section{Surgery}

All surgeries were performed aseptically under $2.5 \%$ isoflurane. The sciatic nerve was exposed at mid-thigh level on the left side of the mice, and then was transected using microdissection scissors. A $3 \mathrm{~mm}$ nerve segment was removed. The two nerve endings were sutured to a $5 \mathrm{~mm}$ SILASTIC silicone tubing (Dow Corning) with 10-0 polypropylene sutures (Ethicon), leaving a 3mm gap in between. Lifeink 200 Hydrogel (highly concentrated type 1 collagen bioinks (Advanced BioMatrix)) mixing with blebbistatin (+/-) $(160 \mu \mathrm{g}$ per mice; Enzo Life Sciences) or vehicle control (6\%DMSO, 44\% saline, 50\% hydrogel) was filled into the gap between two nerve endings with 30-gauge needles (BD). The surgical incisions were closed with 6-0 sutures (Ethicon).

\section{Quantification of axon regeneration in vivo}

After sciatic nerve transection and bridge repair, sciatic nerves were dissected 2, 4 and 8 weeks after injury. Sciatic nerves were fixed and sectioned (details below in Immunohistochemistry section), and regenerating axons were visualized by staining with a chicken anti-Neurofilament $\mathrm{H}$ antibody (Millipore Sigma). Regenerating axons were quantified at specified distances from the injury site using the Sholl analysis plugin of Fiji ImageJ. 


\section{Light and electron microscopic analysis}

Mice were anesthetized with an overdose of ketamine and xylazine $(25 \mathrm{mg} / \mathrm{ml}$ ketamine and $2.5 \mathrm{mg} / \mathrm{ml}$ xylazine in PBS) and a transcardial perfusion was performed with phosphate-buffered saline (PBS), followed by $2 \%$ paraformaldehyde, 2.5\% glutaraldehyde solution (2\%PF, $2.5 \% \mathrm{Glut})$ diluted in phosphate buffer. Sciatic nerves were dissected out and post-fixed in $2 \% \mathrm{PF}$, $2.5 \%$ Glut solution overnight at $4^{\circ} \mathrm{C}$. Next, the tissues were rinsed in PBS and fixed in $1 \%$ Osmium $\left(\mathrm{OsO}_{4}\right)$ solution, washed with $\mathrm{ddH}_{2} \mathrm{O}$ and dehydrated in a series of ethanol and 100\% propylene oxide. The nerves were then embedded in Epon plastic resin, trimmed and cross sectioned $(0.5 \mu \mathrm{m})$, and stained with 1\% toluidine blue solution for light microscopy. The complete sciatic nerve for each subject was photographed at 100X magnification with a Nikon Eclipse E600 microscope coupled with a Nikon camera DS-Fi3 and images automatically stitched together with Nikon NIS-Elements software. To calculate the number of fibers for the Vehicle control and Blebbistatin groups, the full cross section for each nerve was analyzed. The number of fibers was determined based on data segmentation using Neurolucida 360 software (MBF Bioscience). Statistical analysis was performed using the non-parametric Mann-Whitney test (GraphPad Prism, version 8.4.1), and considered significant when the $P$ value was $<0.05$. The data are shown as mean \pm SE. Additional analysis was performed at the electron microscopy level. Ultrathin sections (7090nm) were collected on single-hole formvar-coated grids and counterstained with uranyl acetate and lead citrate. The samples were analyzed under a Tecnai G2 Spirit Twin, FEI®, ThermoFisher Scientific ${ }^{\circledR}$ or Hitachi® H-7500 electron microscopes and images were captured with NanoSprint12 AMT® camera. 


\section{Assessment of motor function recovery}

To assess motor recovery, mice were recorded using a DigiGait apparatus (Mouse Specifics). Mice were forced to walk on a transparent treadmill belt and the digital paw prints were recorded from below using a high-speed digital video camera with the information including print length (PL), toe spread (TS), and intermediate toe spread (IT). The sciatic nerve functional index (SFI) was calculated using the formula: SFI=109.5(ETS-NTS)/NTS-38.3(EPLNPL)/NPL+13.3(EIT-NIT)/NIT-8.8 (Inserra et al., 1998). E is experimental group, $\mathrm{N}$ is normal group. Experiments were conducted fully blinded to drug treatment and surgery.

\section{Immunohistochemistry}

Sciatic nerves were harvested and fixed with 4\% PFA on ice for 1 hour and then cryoprotected with $30 \%$ sucrose in PBS overnight at $4^{\circ} \mathrm{C}$. Sciatic nerves sections $(20 \mu \mathrm{m})$ were collected, blocked and permeabilized with PBS containing $0.3 \%$ Triton $X-100$ and $10 \%$ goat serum (PBTGS) for 1 hour at room temperature (RT). Sections were incubated with a chicken anti-Neurofilament $\mathrm{H}$ antibody (AB5539, Millipore Sigma; 1:1000) diluted in PBTGS overnight at RT and then incubated with Alexa Fluor 488 goat antibody against chicken IgY (A11039, Thermo Fisher; 1:1000) and Hoechst 33342 (H3570, Thermo Fisher; 1:2000) diluted in PBTGS for $1 \mathrm{hr}$ at RT. Images were acquired using a Nikon Eclipse 80I Microscope.

Human induced motor neuron culture were fixed with 4\% PFA for 1 hour, washed with PBS and then permeabilized with PBS containing 0.3\% Triton X100 and 10\% goat serum (PBTGS) for 1 hour at RT. Neurons were then 
incubated with a mouse monoclonal antibody against $\beta$ III-tubulin (T8660, Sigma; 1:1000) diluted in PBTGS overnight, and then were incubated with Alexa Fluor 568 goat anti-mouse IgG (A11031, Thermo Fisher; 1:1000) and Hoechst 33342 (1:2000) diluted in PBTGS for 1 hour at RT. Images were acquired using ArrayScan XTI high-resolution camera (Thermo Fisher).

\section{Caenorhabditis elegans study}

C. elegans strain TU3568 (sid-1(pk3321) him-5(e1490) V; lin-15B(n744) X; uls71 [(pCFJ90) pmyo-2::mCherry + pmec-18::sid-1]) was employed for neuronal-specific RNAi knockdown of the C. elegans homologs of non-muscle myosin II, nmy-1 and nmy-2 (Calixto et al., 2010; Taub et al., 2018). Clones from the Vidal library were streaked onto LB plates containing carbenicillin and grown overnight at $37^{\circ} \mathrm{C}$. Colonies were picked and grown overnight at $37^{\circ} \mathrm{C}$ in $6 \mathrm{~mL}$ of LB supplemented with carbenicillin. $200 \mu \mathrm{L}$ of this subculture was then spread over a NGM agar plate containing 2mM IPTG to induce RNAi expression. A bacterial lawn was grown at room temperature for 3 days. Gravid adult hermaphrodites were bleached and embryos allowed to crawl onto the RNAi-containing bacteria (termed F1s). $30 \mathrm{~F} 1 \mathrm{~s}$ were then picked onto a new RNAi-containing plate and allowed to lay eggs for 4 hours. The resulting embryos (F2s) were examined in regeneration assays at the young adult stage. Regeneration between control RNAi and nmy RNAi were examined.

Axotomy was performed on a Nikon Ti-2000 inverted fluorescent microscope coupled with a Ti:Sapphire infrared laser (Mantis Pulse Switch, Coherent Inc.). Day 1 adults were placed on a 10\% agarose pad and immobilized with a slurry of polystyrene beads (Polysciences). A $1 \mathrm{KHz}$ train of $100 \mathrm{fs}$ pulses (15-30 
$\mathrm{nJ} /$ pulse) was focused through a Nikon 60X 1.4 NA objective to vaporize specifically at the focal point, targeted to be at $20 \mu \mathrm{m}$ and $40 \mu \mathrm{m}$ from the cell body of the Anterior Lateral Microtubule (ALM) neuron, thus creating a gap of $\sim 20 \mu \mathrm{m}$ at the site of injury. This procedure was performed in a span of 15 minutes before returning the adults to the RNAi agar plates for 24 hours. Regeneration was examined by immobilizing the adults with $5 \mathrm{mM}$ sodium azide and capturing a Z-stack a Nikon 40X 1.3NA. A maximum projection image was made and regeneration lengths from the site of injury were measured with FIJl.

\section{Statistical Analysis}

Data were statistically tested with unpaired unequal variances student t-tests. Results in Figure 1, 2 and 6 are expressed as mean \pm standard deviation. Data in Figure 3 and 4 are expressed as the mean \pm standard error of the mean. In the figures, the significance is indicated with asterisks ( ${ }^{*}$ for $p<0.05,{ }^{* \star}$ for $p<0.01$ and $* \star \star$ for $p<0.001$ ). 


\section{Supplemental information}

\section{Supplementary Table 1: Top 20 differentially expressed genes significantly upregulated after blebbistatin treatment (FDR<0.1).}

\begin{tabular}{|c|c|c|c|c|c|}
\hline & Gene & Description & $\begin{array}{l}\text { logFC } \\
\text { Bleb.16h vs } \\
\text { DMSO.16h }\end{array}$ & $\begin{array}{l}\text { logFC } \\
\text { Bleb.26h vs } \\
\text { DMSO.26h }\end{array}$ & $\begin{array}{l}\text { logFC } \\
\text { Bleb.48h vs } \\
\text { DMSO.48h }\end{array}$ \\
\hline 1 & MMP7 & matrix metallopeptidase 7 & 4.388 & 3.047 & 3.765 \\
\hline 2 & BGN & $\begin{array}{l}\text { biglycan: small leucine-rich repeat } \\
\text { proteoglycan (SLRP) found in ECM. }\end{array}$ & 3.589 & 3.565 & 3.204 \\
\hline 3 & TMEM156 & transmembrane protein 156 & 3.263 & 2.518 & 3.177 \\
\hline 4 & CXCL5 & $\begin{array}{l}\text { chemokine ligand } 5 \text {. Neurotrophic effect } \\
\text { to promote neurite outgrowth }\end{array}$ & 3.232 & 3.960 & 4.263 \\
\hline 5 & HABP2 & hyaluronan binding protein 2 & 3.159 & 4.342 & 3.843 \\
\hline 6 & TFPI2 & tissue factor pathway inhibitor 2 & 3.067 & 2.096 & 1.476 \\
\hline 7 & ARSJ & arylsulfatase family, member $\mathrm{J}$ & 3.005 & 3.060 & 3.479 \\
\hline 8 & TFPI & tissue factor pathway inhibitor & 2.993 & 3.057 & 2.862 \\
\hline 9 & FN1 & fibronectin 1 & 2.975 & 3.160 & 3.113 \\
\hline 10 & IFITM2 & $\begin{array}{l}\text { interferon induced transmembrane } \\
\text { protein } 2(1-8 D)\end{array}$ & 2.952 & 3.242 & 1.882 \\
\hline 11 & GBP3 & guanylate binding protein 3 & 2.909 & 1.308 & 2.770 \\
\hline 12 & TCF21 & transcription factor 21 & 2.862 & 1.970 & 2.090 \\
\hline 13 & MYOF & myoferlin & 2.807 & 2.984 & 3.483 \\
\hline 14 & MMP10 & matrix metallopeptidase 10 & 2.731 & 2.938 & 2.120 \\
\hline 15 & TNFSF15 & $\begin{array}{l}\text { tumor necrosis factor (ligand) } \\
\text { superfamily, member } 15\end{array}$ & 2.715 & 2.340 & 4.194 \\
\hline 16 & FAM198B & $\begin{array}{l}\text { family with sequence similarity } 198 \text {, } \\
\text { member B }\end{array}$ & 2.696 & 3.276 & 3.833 \\
\hline
\end{tabular}




\begin{tabular}{|l|l|l|l|l|l|}
\hline 17 & GPR126 & G protein-coupled receptor 126 & 2.695 & 2.262 & 3.066 \\
\hline 18 & CAPN9 & calpain 9 & 2.680 & 3.255 & 2.583 \\
\hline 19 & TACR1 & tachykinin receptor 1 & 2.636 & 3.845 & 4.316 \\
\hline 20 & BMP6 & bone morphogenetic protein 6 & 2.634 & 3.434 & 3.388 \\
\hline
\end{tabular}

Supplementary Figure 1. Dose response curves of two JAK inhibitors. Blebbistatin promotion of outgrowth of human iPSC-derived sensory neurons.

(A-B) Dose response curves of two JAK inhibitors, Baricitinib (A) and Ruxolitinib (B), showing their effects on promoting neurite outgrowth of human motor neurons after a 24 hour treatment. (C-D) The effect of blebbistatin on neurite outgrowth after re-plating on a permissive substrate, laminin. (C) Human iPSCderived sensory neurons; (D) human iPSC-derived motor neurons. Note that human induced sensory neurons did not survive on CSPG after re-plating. Data are represented as mean $\pm S D$. $\left({ }^{\star *} P=0.0027,{ }^{* \star *} P=1.0018 \mathrm{e}-10\right.$, unpaired twotailed t-test).

Supplementary Figure 2. Target validation: knockdown of non-muscle myosin II promotes axon regeneration after injury in human neurons, and in C. elegans. (A-B) Knockdown of non-muscle myosin II with MYH9 shRNA (A) accelerated axon regrowth after injury in human motor neuron spot culture when compared with non-silencing control (B). (C) Knockdown of non-muscle myosin homolog nmy-1 significantly enhances regeneration in C. elegans. 
of human motor neurons. (A-C) Gene ontology analysis of the genes significantly up-regulated in blebbistatin-treated human motor neurons shows enriched molecular functions $16(A), 26(B)$ and 48 hours (C) after blebbistatin vs. DMSO treatment. 


\section{References}

Afshari, F.T., Kappagantula, S., and Fawcett, J.W. (2009). Extrinsic and intrinsic factors controlling axonal regeneration after spinal cord injury. Expert Rev Mol Med 11, e37. Al-Ali, H., Lee, D.H., Danzi, M.C., Nassif, H., Gautam, P., Wennerberg, K., Zuercher, B., Drewry, D.H., Lee, J.K., Lemmon, V.P., et al. (2015). Rational Polypharmacology: Systematically Identifying and Engaging Multiple Drug Targets To Promote Axon Growth. ACS Chem Biol 10, 1939-1951.

Al-Ali, H., Schurer, S.C., Lemmon, V.P., and Bixby, J.L. (2013). Chemical interrogation of the neuronal kinome using a primary cell-based screening assay. ACS Chem Biol 8 , 1027-1036.

Boulting, G.L., Kiskinis, E., Croft, G.F., Amoroso, M.W., Oakley, D.H., Wainger, B.J., Williams, D.J., Kahler, D.J., Yamaki, M., Davidow, L., et al. (2011). A functionally characterized test set of human induced pluripotent stem cells. Nat Biotechnol 29, 279-286.

Burnside, E.R., De Winter, F., Didangelos, A., James, N.D., Andreica, E.C., LayardHorsfall, H., Muir, E.M., Verhaagen, J., and Bradbury, E.J. (2018). Immune-evasive gene switch enables regulated delivery of chondroitinase after spinal cord injury. Brain 141, 2362-2381.

Calixto, A., Chelur, D., Topalidou, I., Chen, X., and Chalfie, M. (2010). Enhanced neuronal RNAi in C. elegans using SID-1. Nat Methods 7, 554-559.

Chan, C.C., Khodarahmi, K., Liu, J., Sutherland, D., Oschipok, L.W., Steeves, J.D., and Tetzlaff, W. (2005). Dose-dependent beneficial and detrimental effects of ROCK inhibitor Y27632 on axonal sprouting and functional recovery after rat spinal cord injury. Exp Neurol 196, 352-364.

Chandran, V., Coppola, G., Nawabi, H., Omura, T., Versano, R., Huebner, E.A., Zhang, A., Costigan, M., Yekkirala, A., Barrett, L., et al. (2016). A Systems-Level Analysis of the Peripheral Nerve Intrinsic Axonal Growth Program. Neuron 89, 956-970. Clarke, K.E., Tams, D.M., Henderson, A.P., Roger, M.F., Whiting, A., and Przyborski, S.A. (2017). A robust and reproducible human pluripotent stem cell derived model of neurite outgrowth in a three-dimensional culture system and its application to study neurite inhibition. Neurochem Int 106, 74-84.

David, S., and Aguayo, A.J. (1981). Axonal elongation into peripheral nervous system "bridges" after central nervous system injury in adult rats. Science 214, 931-933.

Dou, Y., Arlock, P., and Arner, A. (2007). Blebbistatin specifically inhibits actin-myosin interaction in mouse cardiac muscle. Am J Physiol Cell Physiol 293, C1148-1153.

Dupraz, S., Hilton, B.J., Husch, A., Santos, T.E., Coles, C.H., Stern, S., Brakebusch, C., and Bradke, F. (2019). RhoA Controls Axon Extension Independent of Specification in 
the Developing Brain. Curr Biol 29, 3874-3886 e3879.

Eddinger, T.J., Meer, D.P., Miner, A.S., Meehl, J., Rovner, A.S., and Ratz, P.H. (2007). Potent inhibition of arterial smooth muscle tonic contractions by the selective myosin II inhibitor, blebbistatin. J Pharmacol Exp Ther 320, 865-870.

Fagoe, N.D., Attwell, C.L., Kouwenhoven, D., Verhaagen, J., and Mason, M.R. (2015). Overexpression of ATF3 or the combination of ATF3, c-Jun, STAT3 and Smad1 promotes regeneration of the central axon branch of sensory neurons but without synergistic effects. Hum Mol Genet 24, 6788-6800.

Fawcett, J.W. (2020). The Struggle to Make CNS Axons Regenerate: Why Has It Been so Difficult? Neurochem Res 45, 144-158.

Filbin, M.T. (2003). Myelin-associated inhibitors of axonal regeneration in the adult mammalian CNS. Nat Rev Neurosci 4, 703-713.

Fournier, A.E., Takizawa, B.T., and Strittmatter, S.M. (2003). Rho kinase inhibition enhances axonal regeneration in the injured CNS. J Neurosci 23, 1416-1423.

Giger, R.J., Hollis, E.R., 2nd, and Tuszynski, M.H. (2010). Guidance molecules in axon regeneration. Cold Spring Harb Perspect Biol 2, a001867.

Gordon, T., Tyreman, N., and Raji, M.A. (2011). The basis for diminished functional recovery after delayed peripheral nerve repair. J Neurosci 31, 5325-5334.

Grinsell, D., and Keating, C.P. (2014). Peripheral nerve reconstruction after injury: a review of clinical and experimental therapies. Biomed Res Int 2014, 698256. Hiraga, A., Kuwabara, S., Doya, H., Kanai, K., Fujitani, M., Taniguchi, J., Arai, K., Mori, M., Hattori, T., and Yamashita, T. (2006). Rho-kinase inhibition enhances axonal regeneration after peripheral nerve injury. J Peripher Nerv Syst 11, 217-224. Hoffman, P.N. (2010). A conditioning lesion induces changes in gene expression and axonal transport that enhance regeneration by increasing the intrinsic growth state of axons. Exp Neurol 223, 11-18.

Hoke, A., Gordon, T., Zochodne, D.W., and Sulaiman, O.A. (2002). A decline in glial cell-line-derived neurotrophic factor expression is associated with impaired regeneration after long-term Schwann cell denervation. Exp Neurol 173, 77-85. Hu, H.Z., Granger, N., Pai, S.B., Bellamkonda, R.V., and Jeffery, N.D. (2018). Therapeutic efficacy of microtube-embedded chondroitinase $A B C$ in a canine clinical model of spinal cord injury. Brain 141, 1017-1027.

Huebner, E.A., Budel, S., Jiang, Z., Omura, T., Ho, T.S., Barrett, L., Merkel, J.S., Pereira, L.M., Andrews, N.A., Wang, X., et al. (2019). Diltiazem Promotes Regenerative Axon Growth. Mol Neurobiol 56, 3948-3957.

Hur, E.M., Yang, I.H., Kim, D.H., Byun, J., Saijilafu, Xu, W.L., Nicovich, P.R., Cheong, R., Levchenko, A., Thakor, N., et al. (2011). Engineering neuronal growth cones to promote axon regeneration over inhibitory molecules. Proc Natl Acad Sci U S A 108, 
5057-5062.

Inserra, M.M., Bloch, D.A., and Terris, D.J. (1998). Functional indices for sciatic, peroneal, and posterior tibial nerve lesions in the mouse. Microsurgery 18, 119-124. Jenq, C.B., Jenq, L.L., Bear, H.M., and Coggeshall, R.E. (1988). Conditioning lesions of peripheral nerves change regenerated axon numbers. Brain Res 457, 63-69.

Kepiro, M., Varkuti, B.H., Vegner, L., Voros, G., Hegyi, G., Varga, M., and MalnasiCsizmadia, A. (2014). para-Nitroblebbistatin, the non-cytotoxic and photostable myosin II inhibitor. Angew Chem Int Ed Engl 53, 8211-8215.

Klim, J.R., Williams, L.A., Limone, F., Guerra San Juan, I., Davis-Dusenbery, B.N., Mordes, D.A., Burberry, A., Steinbaugh, M.J., Gamage, K.K., Kirchner, R., et al. (2019). ALS-implicated protein TDP-43 sustains levels of STMN2, a mediator of motor neuron growth and repair. Nat Neurosci 22, 167-179.

Koprivica, V., Cho, K.S., Park, J.B., Yiu, G., Atwal, J., Gore, B., Kim, J.A., Lin, E., TessierLavigne, M., Chen, D.F., et al. (2005). EGFR activation mediates inhibition of axon regeneration by myelin and chondroitin sulfate proteoglycans. Science 310, 106-110. Le, A.P., and Friedman, W.J. (2012). Matrix metalloproteinase-7 regulates cleavage of pro-nerve growth factor and is neuroprotective following kainic acid-induced seizures. J Neurosci 32, 703-712.

Lee, R., Kermani, P., Teng, K.K., and Hempstead, B.L. (2001). Regulation of cell survival by secreted proneurotrophins. Science 294, 1945-1948.

Li, H., Kuwajima, T., Oakley, D., Nikulina, E., Hou, J., Yang, W.S., Lowry, E.R., Lamas, N.J., Amoroso, M.W., Croft, G.F., et al. (2016). Protein Prenylation Constitutes an Endogenous Brake on Axonal Growth. Cell Rep 16, 545-558. Limouze, J., Straight, A.F., Mitchison, T., and Sellers, J.R. (2004). Specificity of blebbistatin, an inhibitor of myosin II. J Muscle Res Cell Motil 25, 337-341. Lingor, P., Tonges, L., Pieper, N., Bermel, C., Barski, E., Planchamp, V., and Bahr, M. (2008). ROCK inhibition and CNTF interact on intrinsic signalling pathways and differentially regulate survival and regeneration in retinal ganglion cells. Brain 131, 250-263.

Lundborg, G. (2000). A 25-year perspective of peripheral nerve surgery: evolving neuroscientific concepts and clinical significance. J Hand Surg Am 25, 391-414. Ma, C.H., Omura, T., Cobos, E.J., Latremoliere, A., Ghasemlou, N., Brenner, G.J., van Veen, E., Barrett, L., Sawada, T., Gao, F., et al. (2011). Accelerating axonal growth promotes motor recovery after peripheral nerve injury in mice. J Clin Invest 121, 4332-4347.

Ma, T.C., Campana, A., Lange, P.S., Lee, H.H., Banerjee, K., Bryson, J.B., Mahishi, L., Alam, S., Giger, R.J., Barnes, S., et al. (2010). A large-scale chemical screen for regulators of the arginase 1 promoter identifies the soy isoflavone daidzeinas a 
clinically approved small molecule that can promote neuronal protection or regeneration via a cAMP-independent pathway. J Neurosci 30, 739-748.

Mar, F.M., Bonni, A., and Sousa, M.M. (2014). Cell intrinsic control of axon regeneration. EMBO Rep 15, 254-263.

McQuarrie, I.G., Grafstein, B., and Gershon, M.D. (1977). Axonal regeneration in the rat sciatic nerve: effect of a conditioning lesion and of dbcAMP. Brain Res 132, 443453.

Neumann, S., and Woolf, C.J. (1999). Regeneration of dorsal column fibers into and beyond the lesion site following adult spinal cord injury. Neuron 23, 83-91. Novak, C.B., Anastakis, D.J., Beaton, D.E., Mackinnon, S.E., and Katz, J. (2011). Biomedical and psychosocial factors associated with disability after peripheral nerve injury. J Bone Joint Surg Am 93, 929-936.

Renthal, W., Tochitsky, I., Yang, L., Cheng, Y.C., Li, E., Kawaguchi, R., Geschwind, D.H., and Woolf, C.J. (2020). Transcriptional Reprogramming of Distinct Peripheral Sensory Neuron Subtypes after Axonal Injury. Neuron 108, 128-144 e129.

Richardson, P.M., McGuinness, U.M., and Aguayo, A.J. (1980). Axons from CNS neurons regenerate into PNS grafts. Nature 284, 264-265.

Roman, B.I., Verhasselt, S., and Stevens, C.V. (2018). Medicinal Chemistry and Use of Myosin II Inhibitor ( S)-Blebbistatin and Its Derivatives. J Med Chem 61, 9410-9428. Rosner, H., Moller, W., Wassermann, T., Mihatsch, J., and Blum, M. (2007). Attenuation of actinomyosinll contractile activity in growth cones accelerates filopodia-guided and microtubule-based neurite elongation. Brain Res 1176, 1-10. Schindelin, J., Arganda-Carreras, I., Frise, E., Kaynig, V., Longair, M., Pietzsch, T., Preibisch, S., Rueden, C., Saalfeld, S., Schmid, B., et al. (2012). Fiji: an open-source platform for biological-image analysis. Nat Methods 9, 676-682.

Schwab, M.E., and Strittmatter, S.M. (2014). Nogo limits neural plasticity and recovery from injury. Curr Opin Neurobiol 27, 53-60.

Seijffers, R., Mills, C.D., and Woolf, C.J. (2007). ATF3 increases the intrinsic growth state of DRG neurons to enhance peripheral nerve regeneration. J Neurosci 27, 79117920.

Sherman, S.A., Phillips, J.K., Costa, J.T., Cho, F.S., Oungoulian, S.R., and Finan, J.D. (2016). Stretch Injury of Human Induced Pluripotent Stem Cell Derived Neurons in a 96 Well Format. Sci Rep 6, 34097.

Sherman, S.P., and Bang, A.G. (2018). High-throughput screen for compounds that modulate neurite growth of human induced pluripotent stem cell-derived neurons. Dis Model Mech 11.

Songcharoen, P., Wongtrakul, S., Mahaisavariya, B., and Spinner, R.J. (2001). Hemicontralateral $\mathrm{C} 7$ transfer to median nerve in the treatment of root avulsion brachial 
plexus injury. J Hand Surg Am 26, 1058-1064.

Straight, A.F., Cheung, A., Limouze, J., Chen, I., Westwood, N.J., Sellers, J.R., and Mitchison, T.J. (2003). Dissecting temporal and spatial control of cytokinesis with a myosin II Inhibitor. Science 299, 1743-1747.

Taub, D.G., Awal, M.R., and Gabel, C.V. (2018). O-GIcNAc Signaling Orchestrates the Regenerative Response to Neuronal Injury in Caenorhabditis elegans. Cell Rep 24, 1931-1938 e1933.

Tran, A.P., Warren, P.M., and Silver, J. (2018). The Biology of Regeneration Failure and Success After Spinal Cord Injury. Physiol Rev 98, 881-917.

Usher, L.C., Johnstone, A., Erturk, A., Hu, Y., Strikis, D., Wanner, I.B., Moorman, S., Lee, J.W., Min, J., Ha, H.H., et al. (2010). A chemical screen identifies novel compounds that overcome glial-mediated inhibition of neuronal regeneration. $J$ Neurosci 30, 4693-4706.

Varkuti, B.H., Kepiro, M., Horvath, I.A., Vegner, L., Rati, S., Zsigmond, A., Hegyi, G., Lenkei, Z., Varga, M., and Malnasi-Csizmadia, A. (2016). A highly soluble, nonphototoxic, non-fluorescent blebbistatin derivative. Sci Rep 6, 26141.

Walker, A., Su, H., Conti, M.A., Harb, N., Adelstein, R.S., and Sato, N. (2010). Nonmuscle myosin II regulates survival threshold of pluripotent stem cells. Nat Commun 1,71 .

Wang, H.H., Tanaka, H., Qin, X., Zhao, T., Ye, L.H., Okagaki, T., Katayama, T., Nakamura, A., Ishikawa, R., Thatcher, S.E., et al. (2008). Blebbistatin inhibits the chemotaxis of vascular smooth muscle cells by disrupting the myosin II-actin interaction. Am J Physiol Heart Circ Physiol 294, H2060-2068.

Wang, P., Gorter, R.P., de Jonge, J.C., Nazmuddin, M., Zhao, C., Amor, S., Hoekstra, D., and Baron, W. (2018). MMP7 cleaves remyelination-impairing fibronectin aggregates and its expression is reduced in chronic multiple sclerosis lesions. Glia 66, 1625-1643. Wang, X., Yigitkanli, K., Kim, C.Y., Sekine-Komo, T., Wirak, D., Frieden, E., Bhargava, A., Maynard, G., Cafferty, W.B., and Strittmatter, S.M. (2014). Human NgR-Fc decoy protein via lumbar intrathecal bolus administration enhances recovery from rat spinal cord contusion. J Neurotrauma 31, 1955-1966.

Wells, J.E., Rice, T.K., Nuttall, R.K., Edwards, D.R., Zekki, H., Rivest, S., and Yong, V.W. (2003). An adverse role for matrix metalloproteinase 12 after spinal cord injury in mice. J Neurosci 23, 10107-10115.

Yiu, G., and He, Z. (2006). Glial inhibition of CNS axon regeneration. Nat Rev Neurosci 7, 617-627.

Young, E.J., Blouin, A.M., Briggs, S.B., Sillivan, S.E., Lin, L., Cameron, M.D., Rumbaugh, G., and Miller, C.A. (2016). Nonmuscle myosin IIB as a therapeutic target for the prevention of relapse to methamphetamine use. Mol Psychiatry 21, 615-623. 
Zhang, H., Yang, R., Wang, Z., Lin, G., Lue, T.F., and Lin, C.S. (2011). Adipose tissuederived stem cells secrete CXCL5 cytokine with neurotrophic effects on cavernous nerve regeneration. J Sex Med 8, 437-446.

Zhong, J., and Zou, H. (2014). BMP signaling in axon regeneration. Curr Opin Neurobiol 27, 127-134. 
(neurite outgrowth after replating)

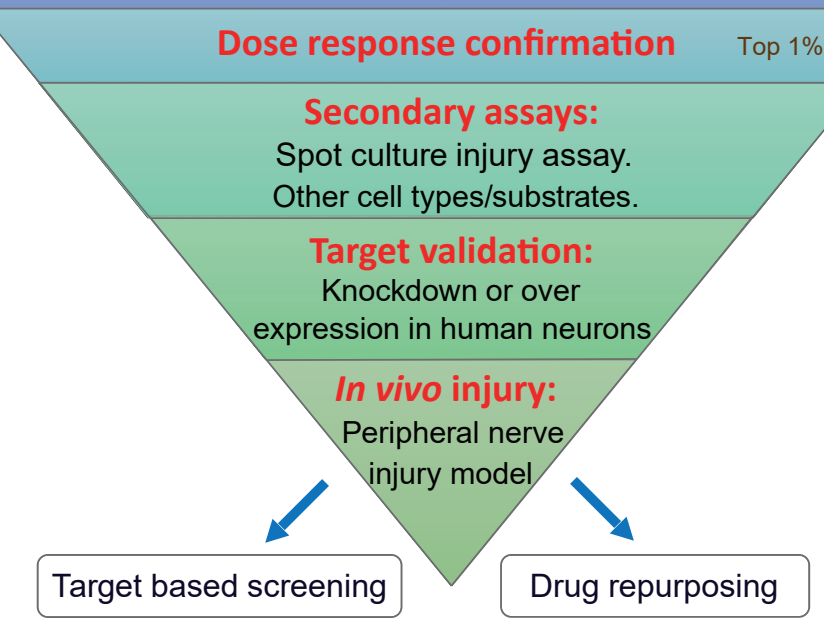

$4557 \mathrm{cpds}$

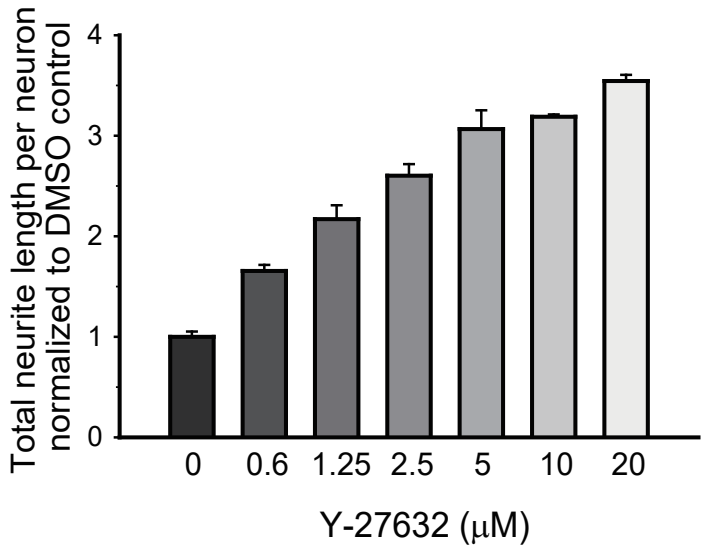

C Laminin

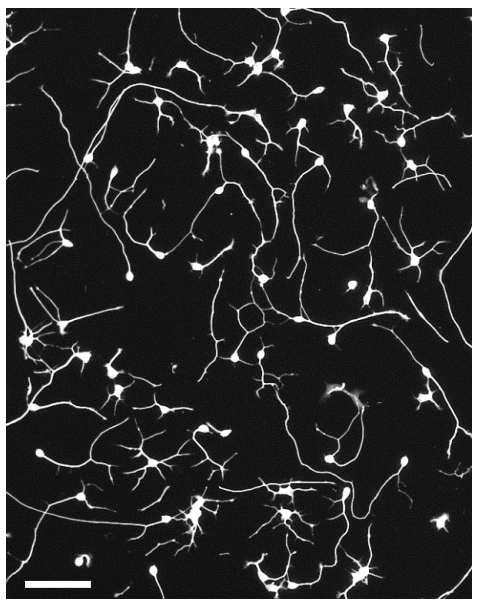

G

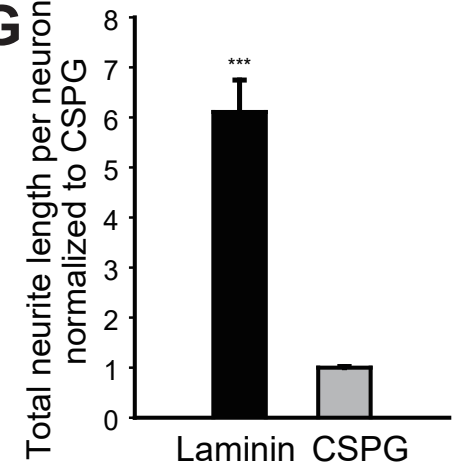

$J$

J Plate hES/iPS Day $\frac{1}{-2}$
D

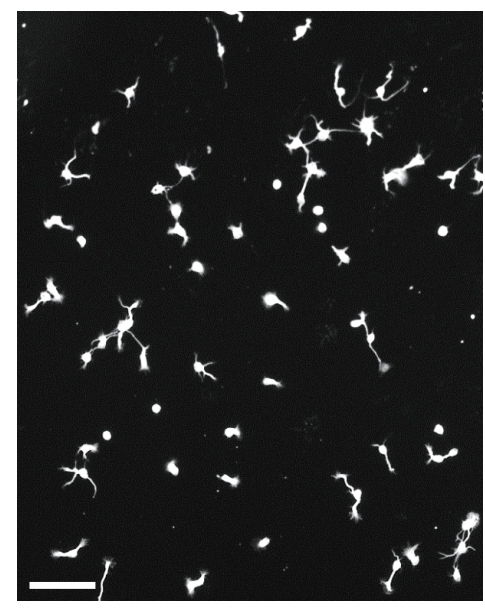

H

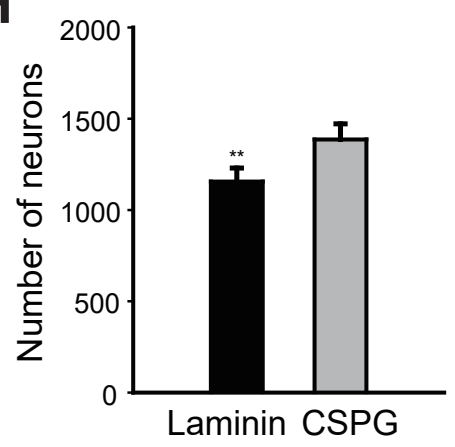

E

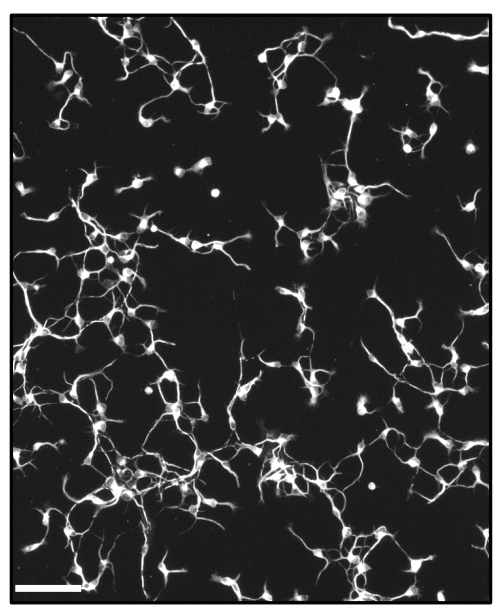

F DMSO

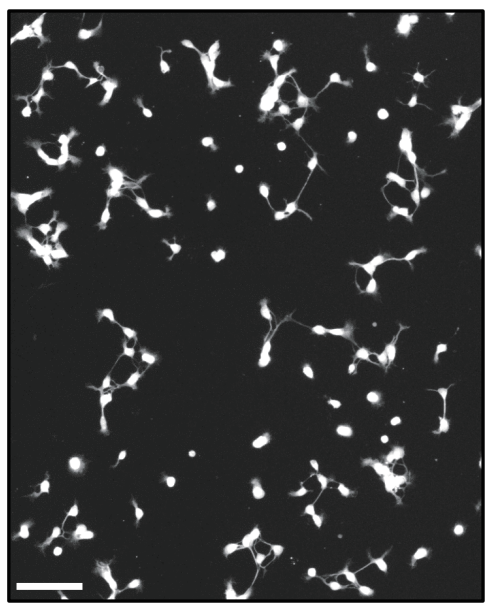

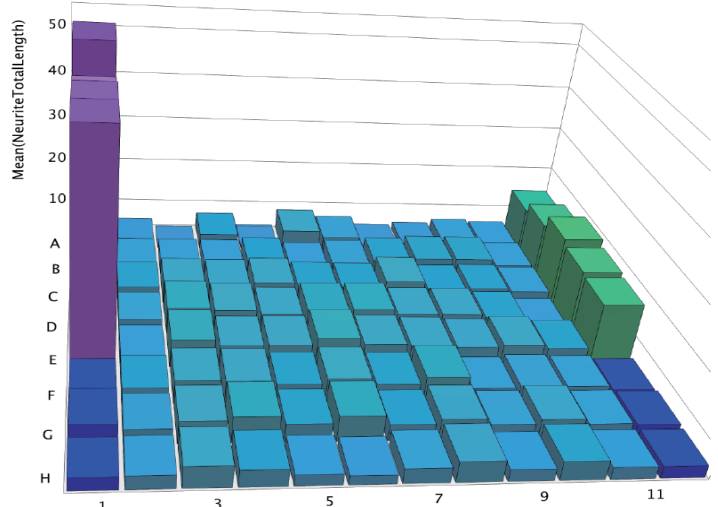

$\mathrm{K}$

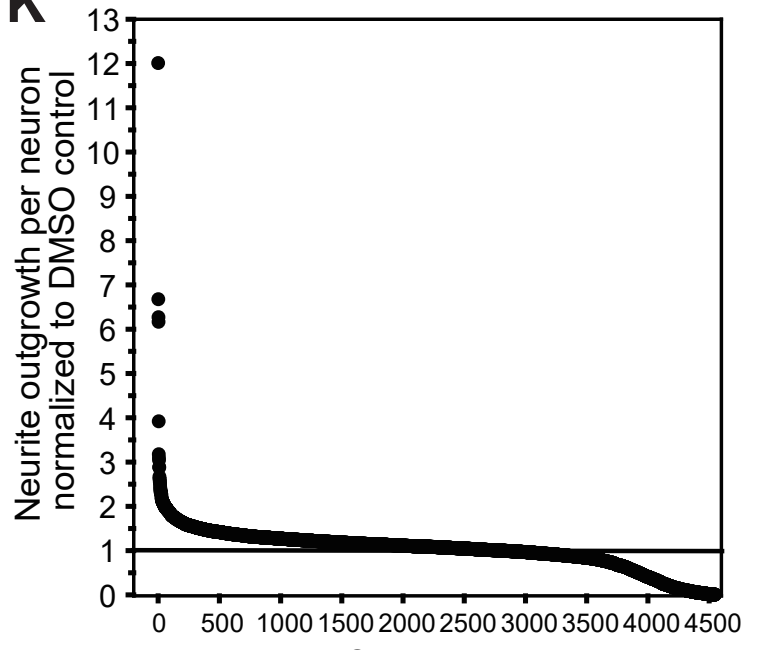

Compounds 

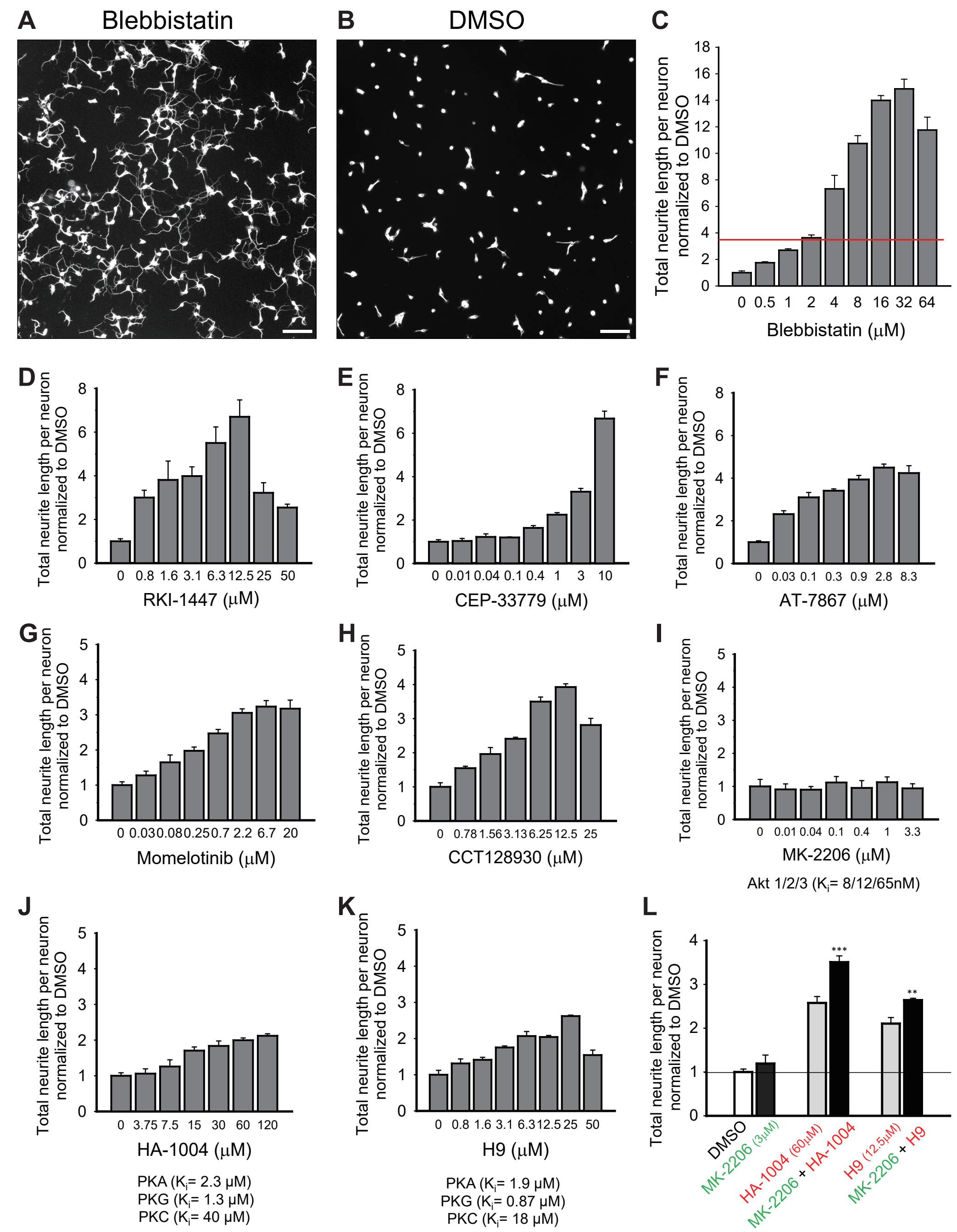

Akt $1 / 2 / 3\left(K_{i}=8 / 12 / 65 n M\right)$

L

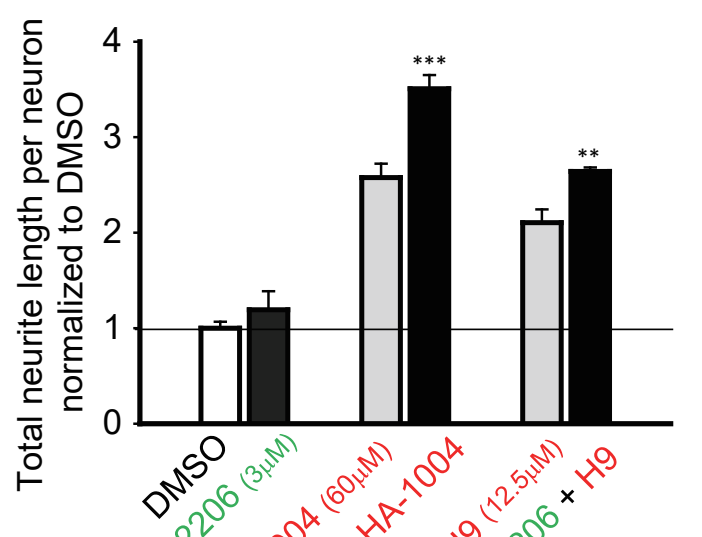

PKA $\left(\mathrm{K}_{\mathrm{K}}=1.9 \mu \mathrm{M}\right)$ PKC $\left(K_{i}=18 \mu \mathrm{M}\right)$ 
Day 1

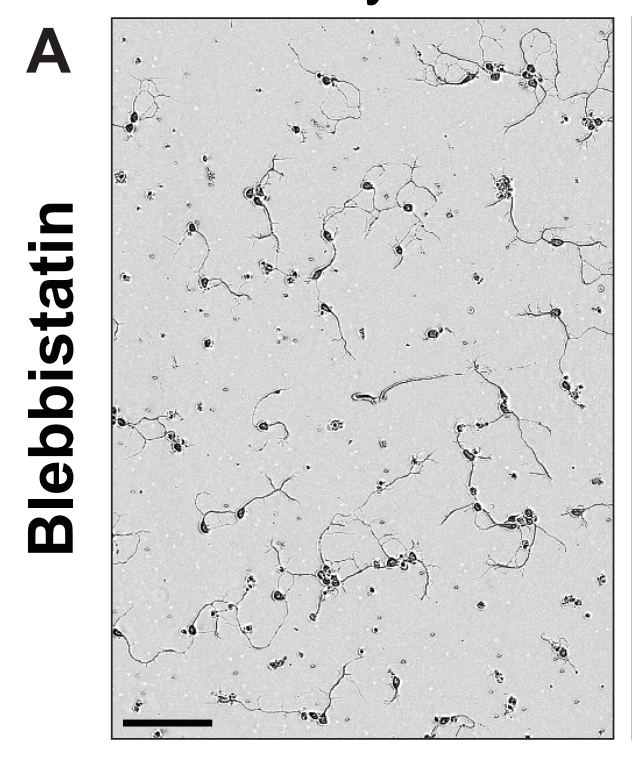

B
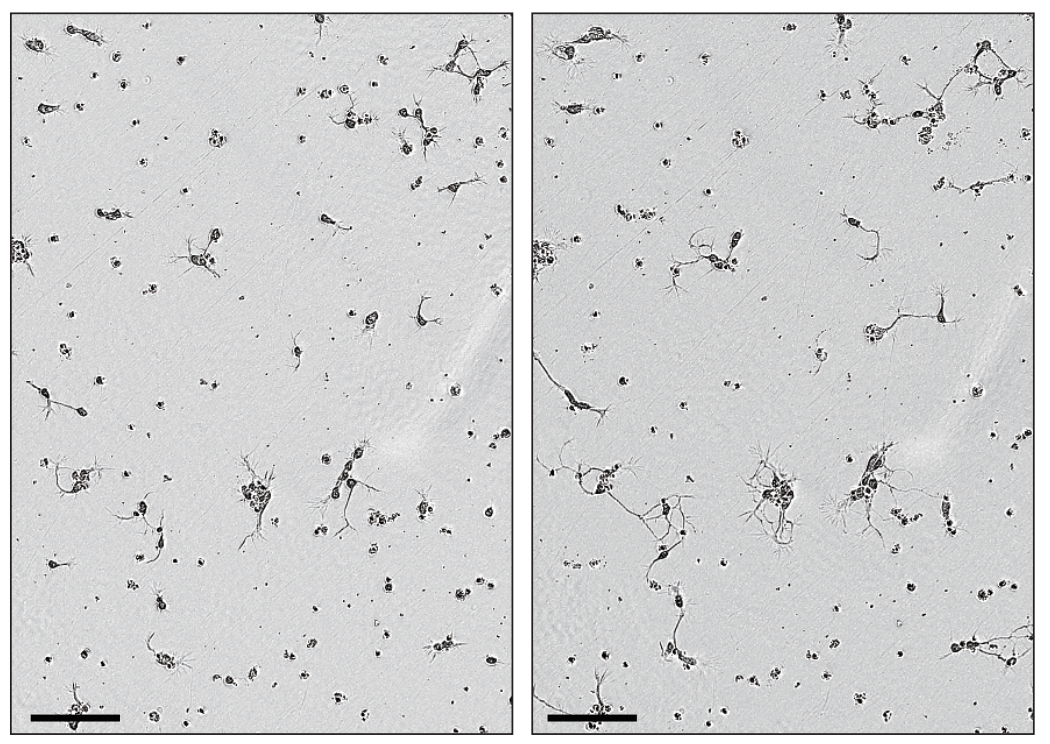

C

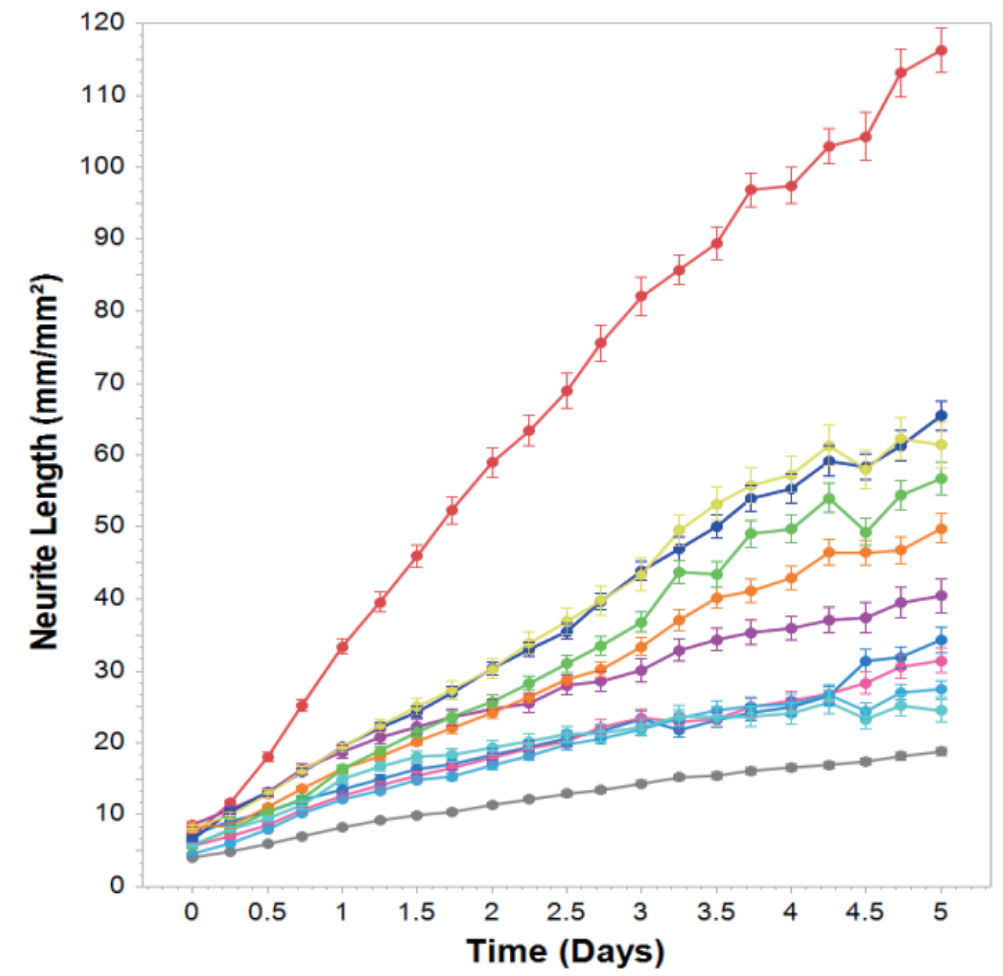

Day 5
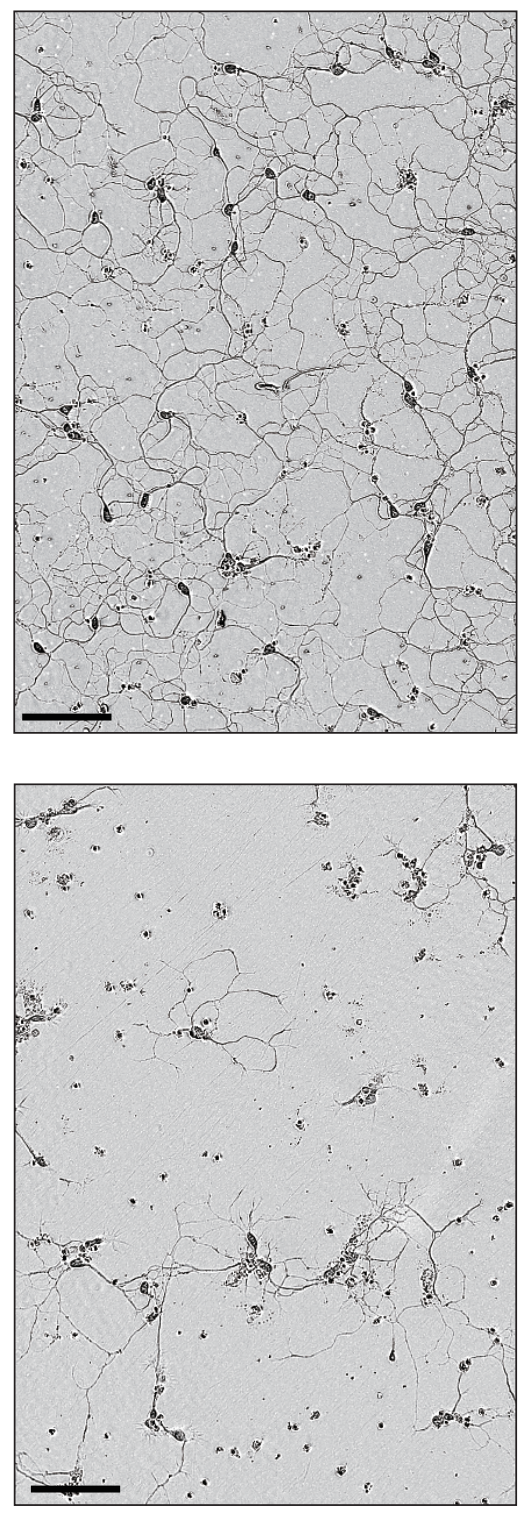

\section{- DMSO}

- Momelotinib 10uM

- CCT128930 10uM

- Blebbistatin 25uM

- Thiazovivin 50uM

- RKI-1447 15uM

- Y-27632 20uM

- CEP-33779 10uM

- Benidipine hydrochloride 10uM - AT7867 10uM HA1077 (Fasudil) 10uM 
A

human iPSCs motor neurons spot culture laser anatomy (+ drug) analysis
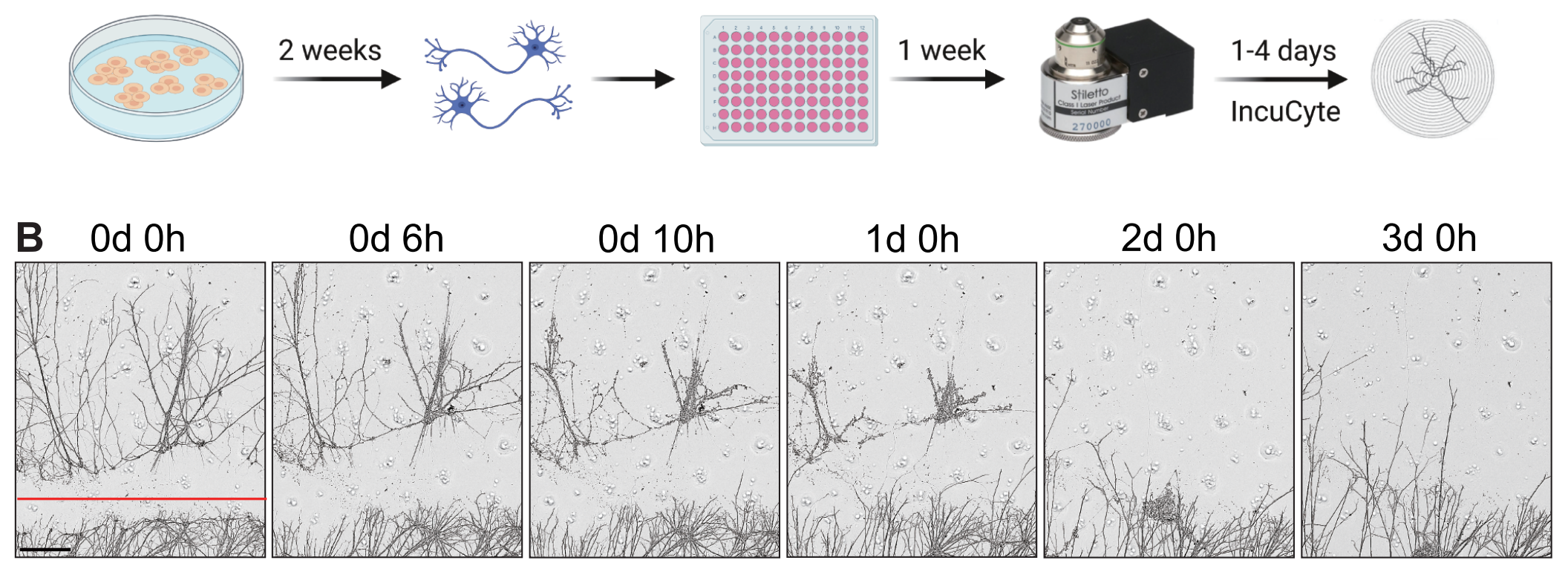

Cell bodies
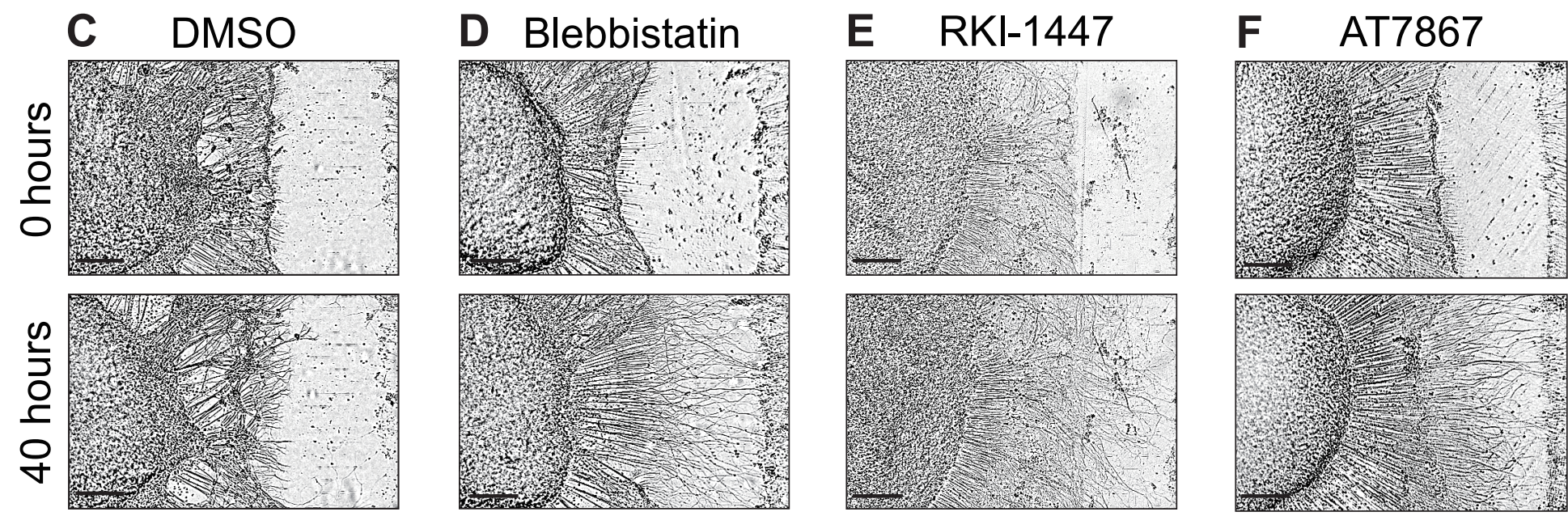

G
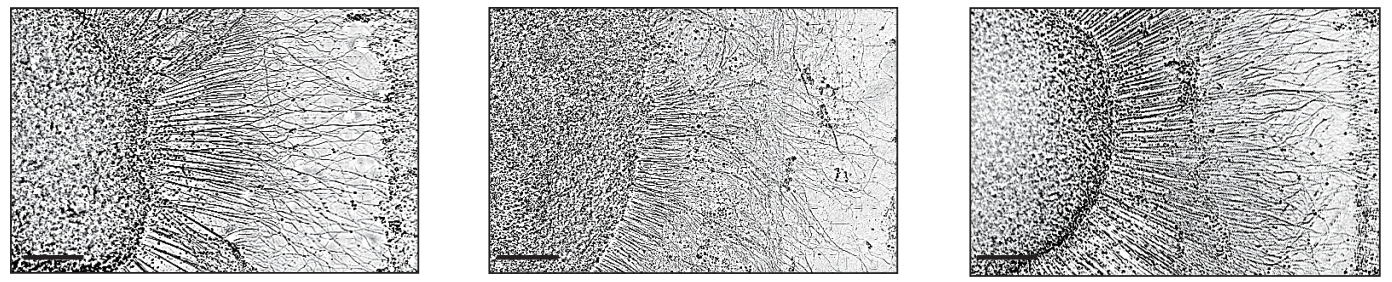

H

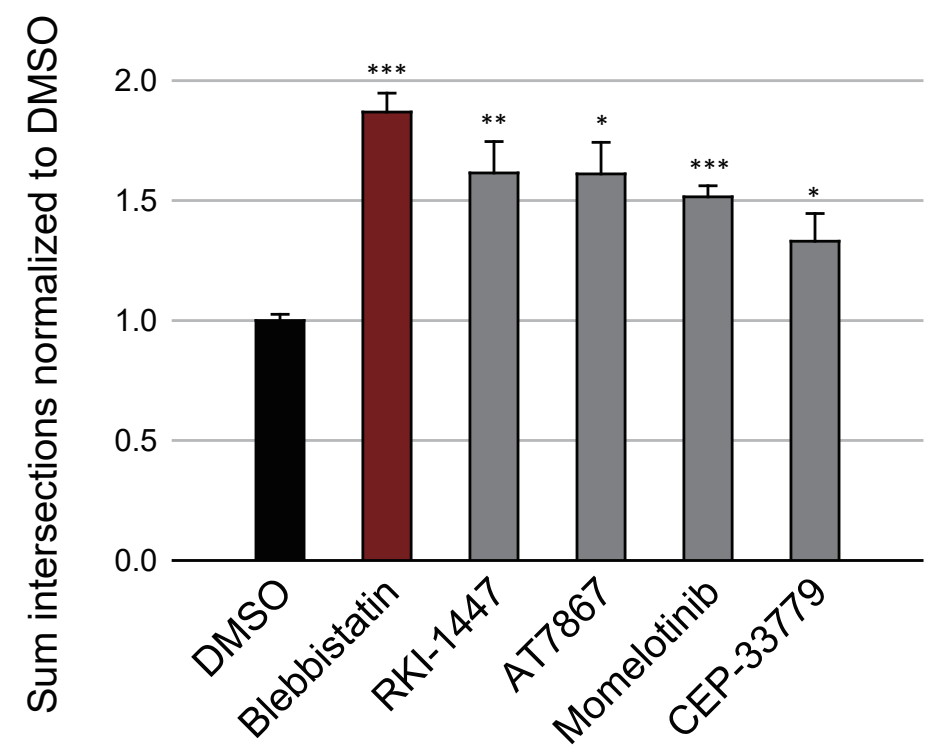




\section{A Bleb vs DMSO 16hr}

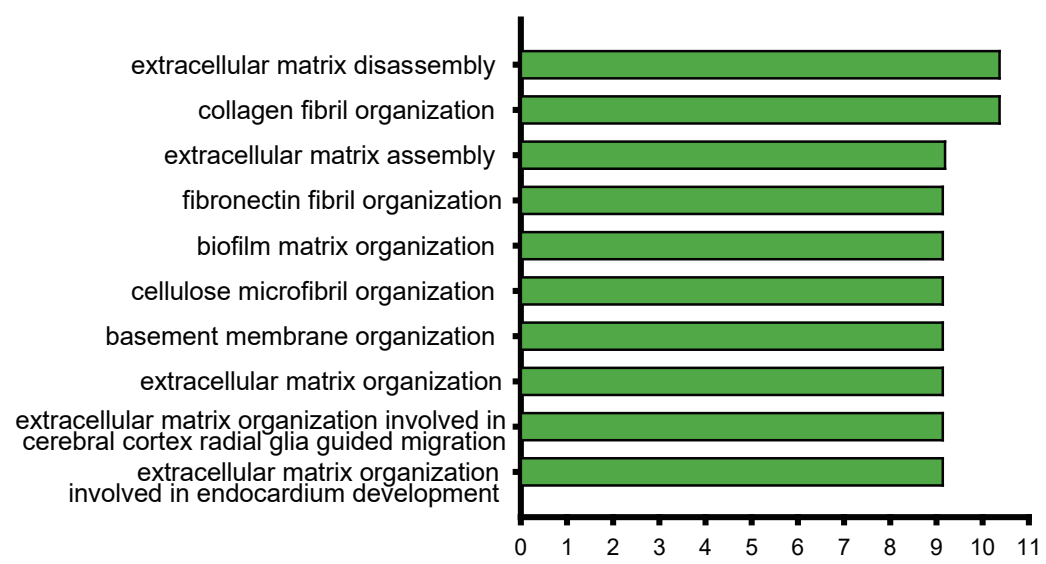

GO Biological Process (-Log10(Adjusted P-value))

\section{B Bleb vs DMSO 26hr}

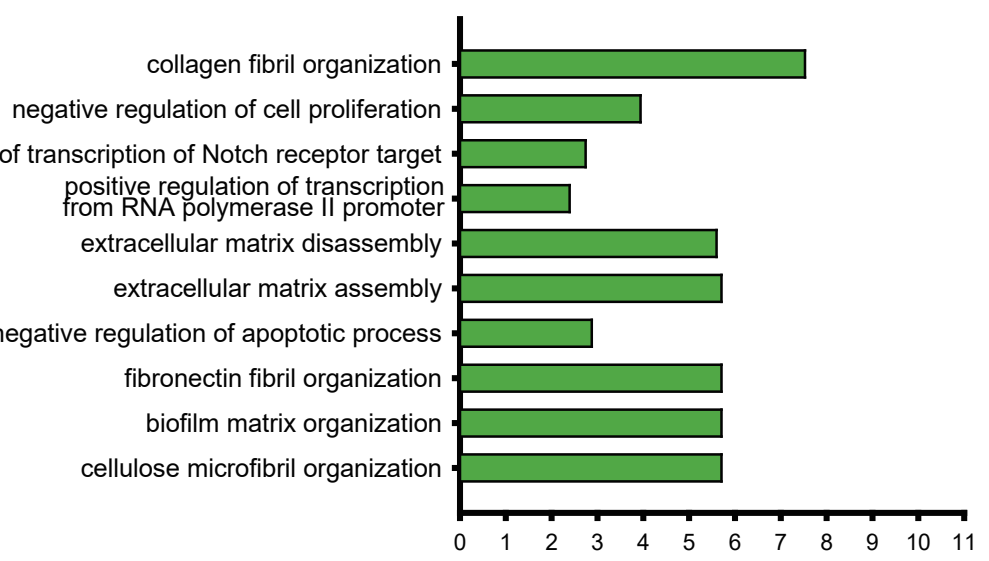

GO Biological Process (-Log10(Adjusted P-value))

\section{Bleb vs DMSO 48hr}

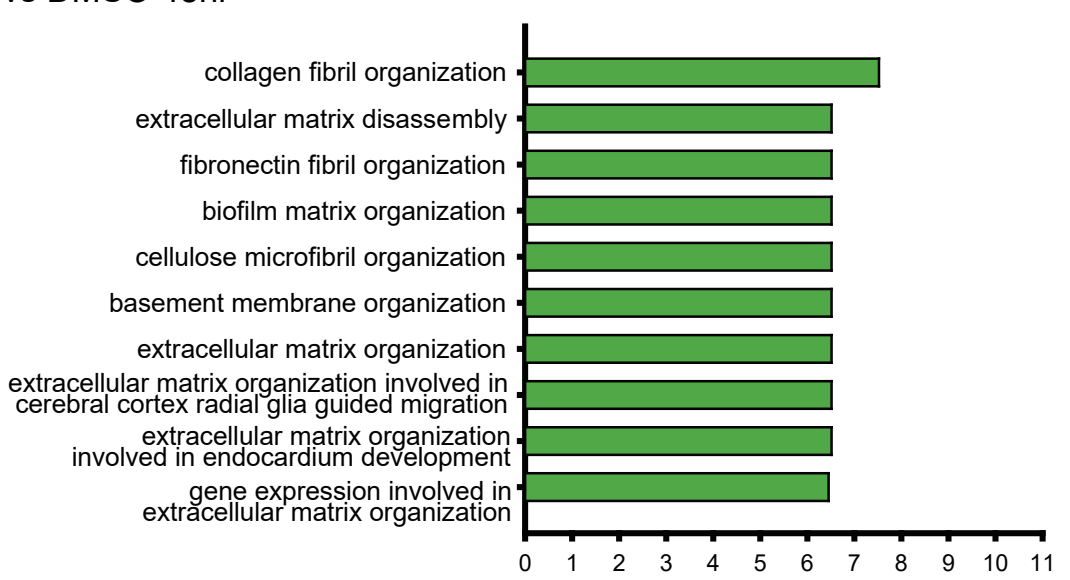

GO Biological Process (-Log10(Adjusted P-value))

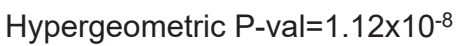

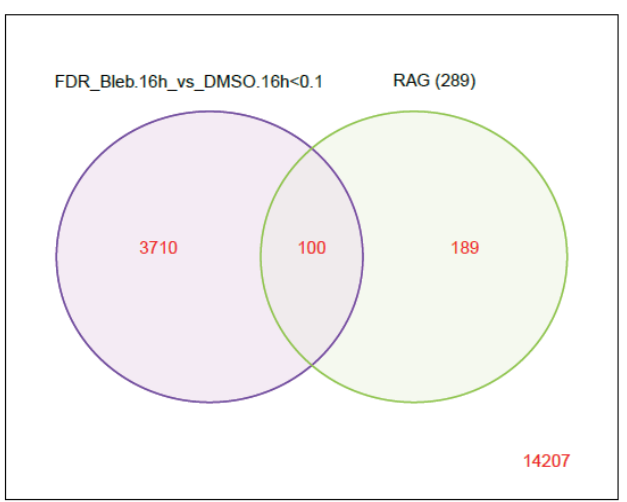

E

Hypergeometric $\mathrm{P}$-val=5.38 $\times 10^{-11}$

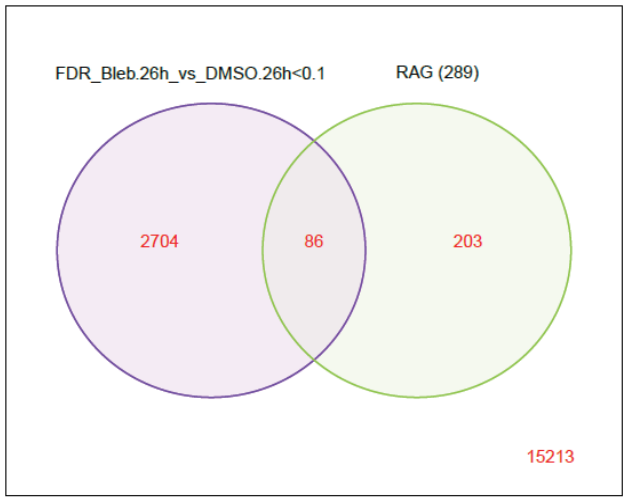

$\mathbf{F}$

Hypergeometric P-val=3.16x10-11

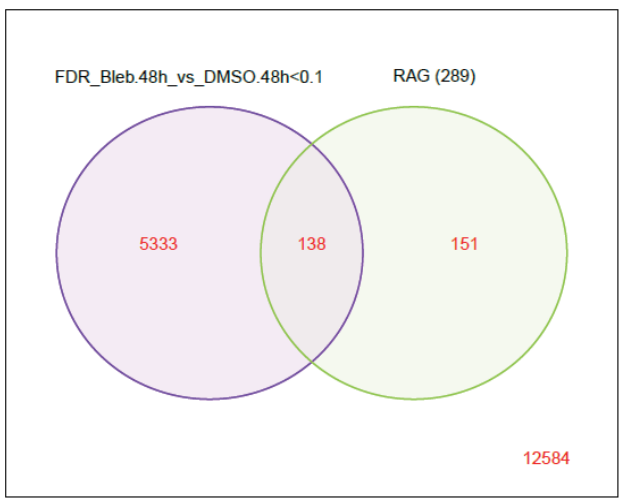

Color Key and Histogram

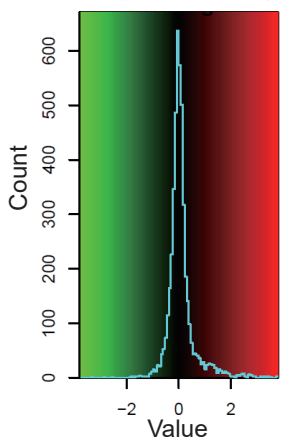

Bleb 16h vs DMSO 16h

Bleb 26h vs DMSO 26h

Bleb $48 \mathrm{~h}$ vs DMSO $48 \mathrm{~h}$

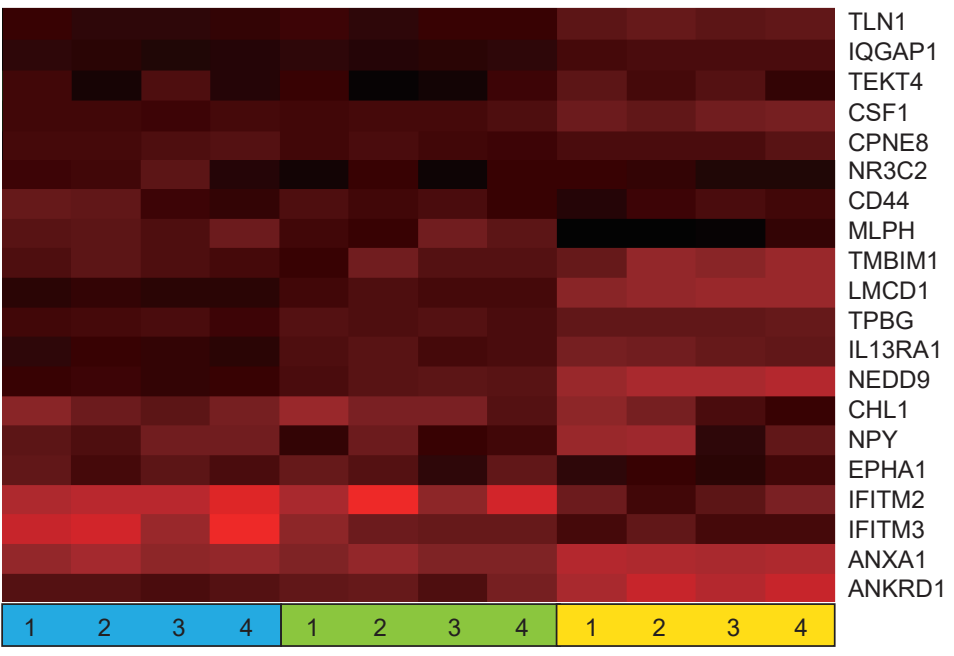


A
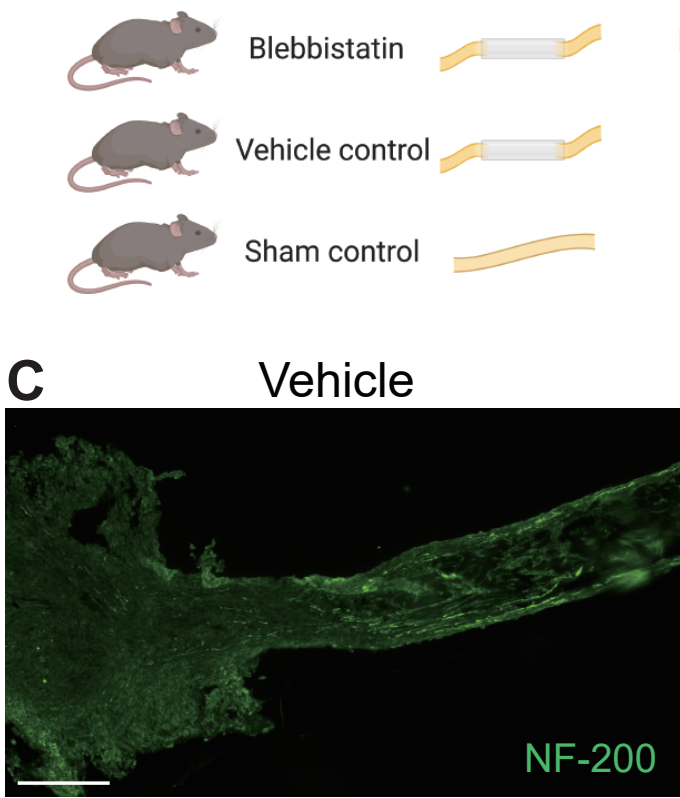

proximal

distal

D
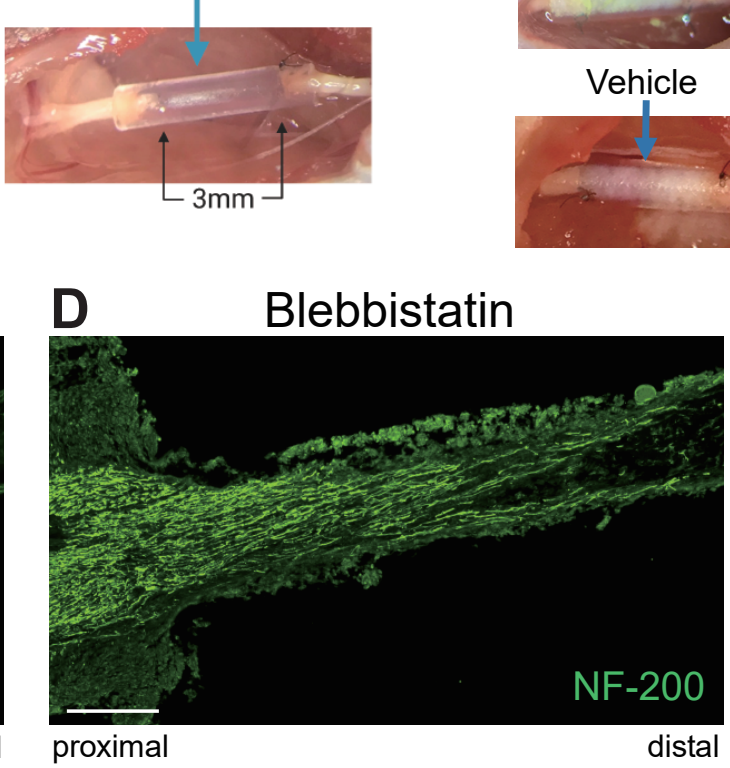

B

Blebbistatin

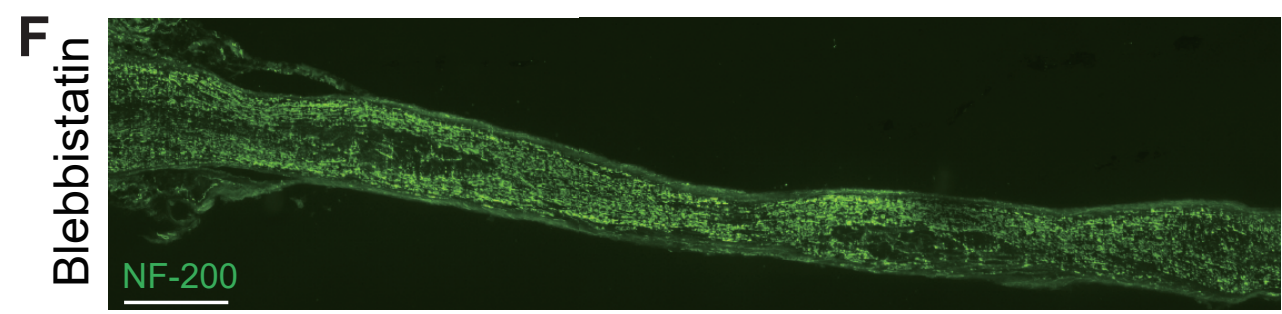

G

$$
\frac{\frac{0}{0}}{\frac{0}{\frac{0}{0}}}
$$

H $\frac{\mathrm{NF}-200}{\text { proximal }}$
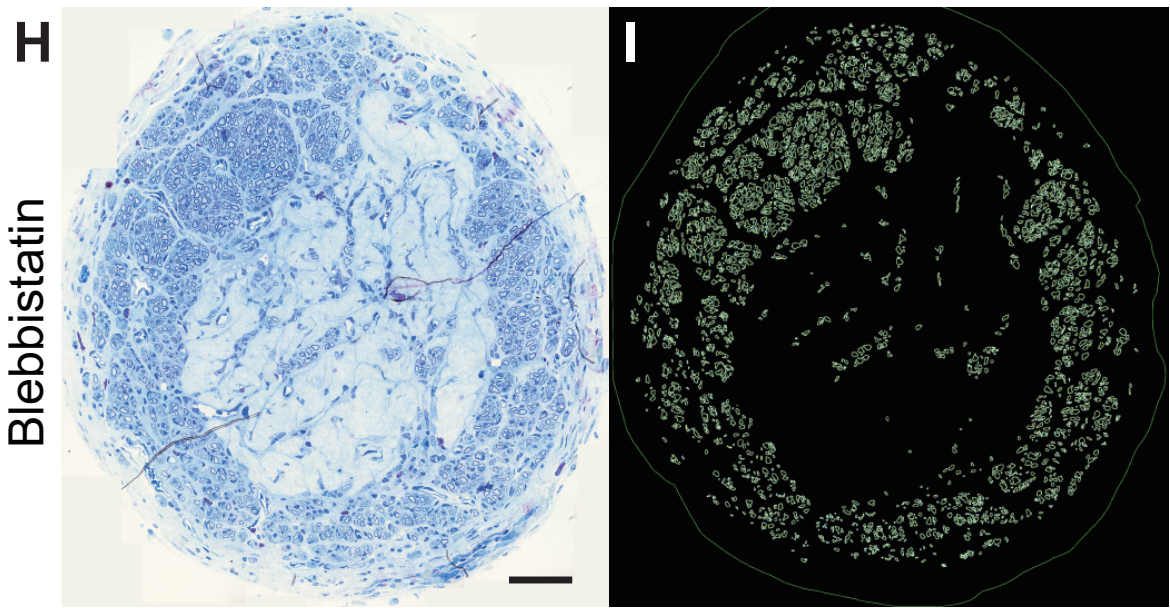

$J$

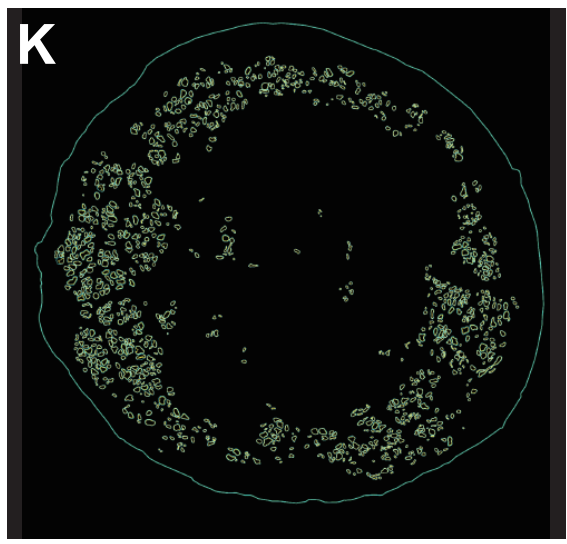

distal

L.
$\frac{\pi}{\pi}$
$\frac{0}{0}$
$\frac{0}{0}$
$\frac{0}{0}$
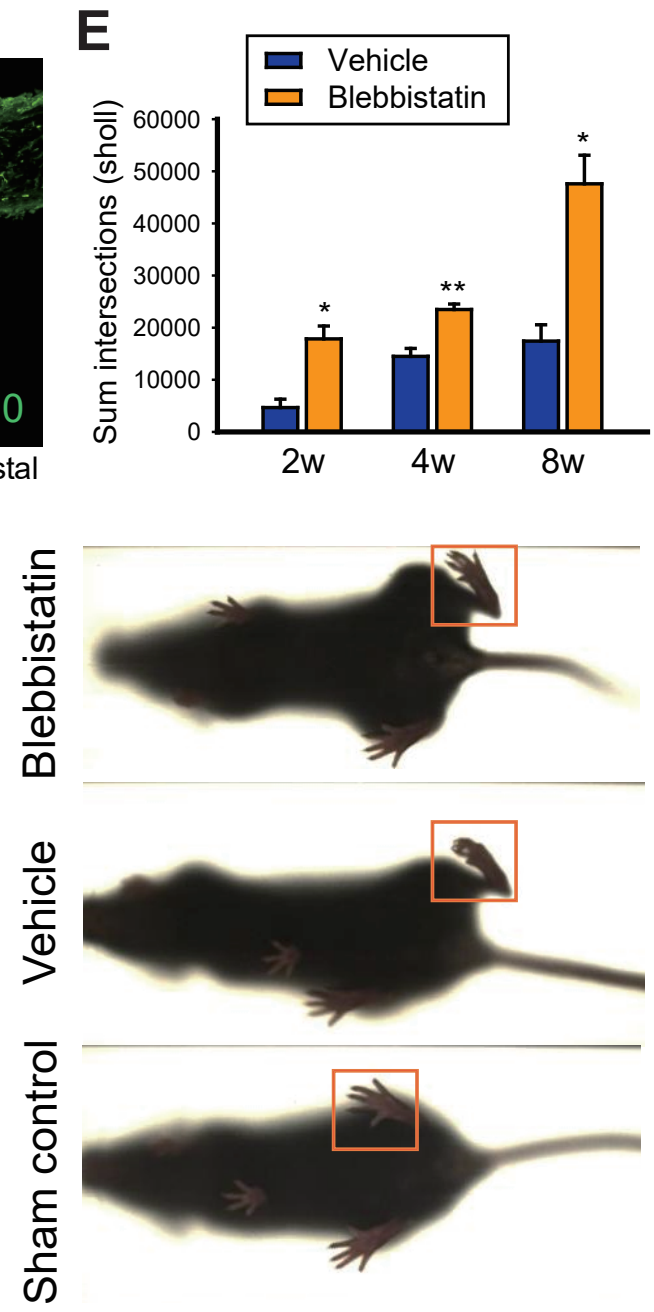

M

$\mathbf{N}$
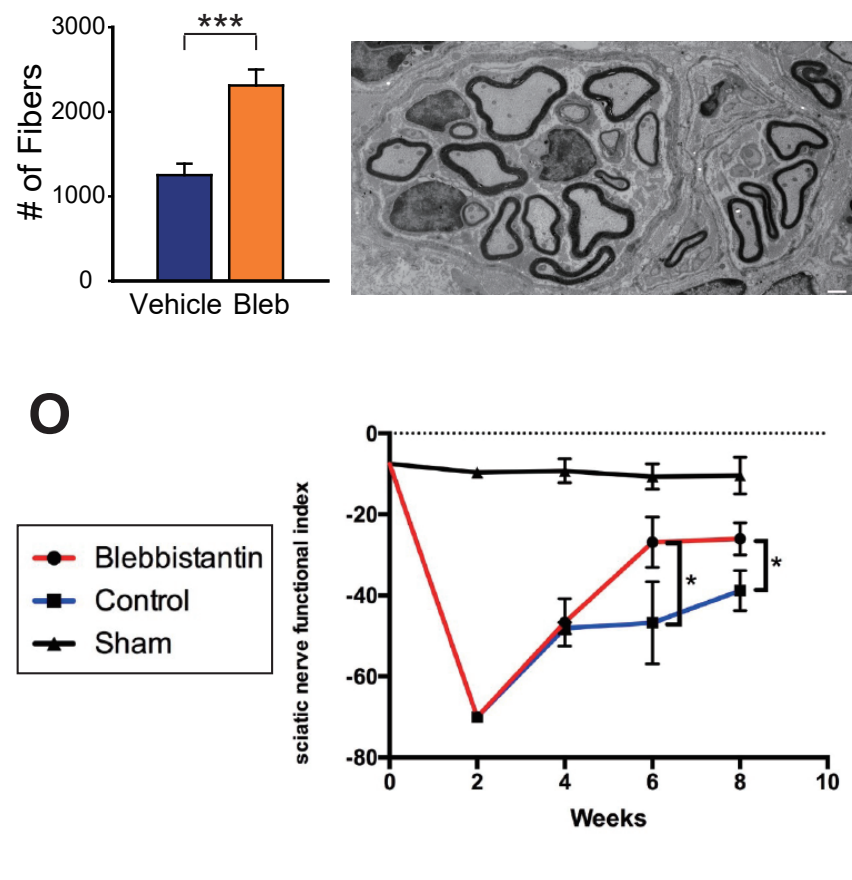